The Astrophysical Journal, 305:698-713, 1986 June 15

(C) 1986. The American Astronomical Society. All rights reserved. Printed in U.S.A.

\title{
THE C, N, AND O ABUNDANCES OF GIANT STARS IN $\omega$ CENTAURI $^{1}$
}

\author{
J. G. COHEN \\ California Institute of Technology \\ AND \\ R. A. BELL \\ Astronomy Program, University of Maryland \\ Received 1985 August 9; accepted 1985 December 4
}

\begin{abstract}
Indices measuring the strength of $\mathrm{CH}, \mathrm{C}_{2}, \mathrm{CN}$, and $\mathrm{NH}$ bands and $\mathrm{Ca}$ II and $\mathrm{Ca}$ I lines have been determined for 72 stars in $\omega$ Cen. These data, and narrow-band photometry of the $2.4 \mu \mathrm{m} \mathrm{CO}$ bands, are calibrated by using theoretical indices derived from synthetic spectra which are computed via a grid of model atmospheres of appropriate parameters.

In addition to the four $\mathrm{C}$ stars, we find two groups of giants in $\omega$ Cen. Both groups include stars spanning a wide range of abundances of the heavy elements, $Z$. The $N$-enhanced stars have $N / Z$ enhanced by a factor of 5 to 15 , while both $\mathrm{C} / Z$ and $\mathrm{O} / Z$ are depleted by a factor which may be as much as 10 . This depletion allows the $\mathrm{CH}$ and $\mathrm{CO}$ features to be fitted satisfactorily and yields enough nitrogen to fit $\mathrm{CN}$ and $\mathrm{NH}$ features. The $\mathrm{N}$-normal stars have $\mathrm{N} / Z$ and $\mathrm{C} / Z$ within factor of 2 of solar. The range of variation of $\mathrm{C}$ and $\mathrm{N}$ is typical of those seen in other globular clusters.
\end{abstract}

Subject headings: clusters: globular — stars: abundances — stars: evolution — stars: late-type

\section{INTRODUCTION}

It is well established that the globular cluster $\omega$ Cen is unique among the galactic globulars. It possesses a wide giant branch in the luminosity-effective temperature plane (see, for example, Woolley et al. 1966 or Cannon and Stobie 1973). Spectra of individual giants (Cohen 1981; Mallia and Pagel 1981; Gratton 1982), of RR Lyrae variables (Freeman and Rodgers 1975), and of turn-off region stars (Hesser et al. 1985) have all demonstrated that there is a wide range in metal abundance among the $\omega$ Cen stars. Persson et al. (1980) (henceforth PFCAM) showed that there is a wide range in strength of the $2.3 \mu \mathrm{m} \mathrm{CO}$ band at a given $V-K$ color (i.e. a given $T_{\text {eff }}$ ).

The purpose of this paper is to explore the abundances of the light elements $\mathrm{C}, \mathrm{N}$, and $\mathrm{O}$ in the $\omega$ Cen giants and to compare the results with theoretical predictions and with the results for stars in other extensively studied globular clusters which are uniform in heavy element abundances. The observational data are presented in $\S$ II. Correlations among the measured indices for various molecular and atomic absorption features are described in $\S$ III, while $\S$ IV presents a calibration of the observed indices derived from spectral synthesis techniques applied to model atmospheres of varying $T_{\text {eff }}$, gravity, metallicity, and $\mathrm{C}, \mathrm{N}$, and $\mathrm{O}$ abundances. The implications of the variations in light element abundances are discussed in $\S \mathrm{V}$. A summary of the important conclusions is presented in $\S \mathrm{VI}$.

\section{OBSERVATIONS}

A sample of 82 stars on the upper giant branch (GB) of $\omega$ Centauri was studied in the infrared by PFCAM. Ignoring the large amplitude variables, we have observed all the stars on their list with measured $\mathrm{CO}$ indices, plus the brighter of those without $\mathrm{CO}$ indices, to form the sample of 72 program stars.

\footnotetext{
${ }^{1}$ Observations were made at Las Campanas Observatory as part of a collaborative agreement between the California Institute of Technology and the Carnegie Institution of Washington.
}

Radial velocity measurements suggest that all these stars are members. These observations were made with the intensified Reticon detector (Shectman 1978) at the Cassegrain focus of the $2.5 \mathrm{~m}$ Du Pont telescope of the Las Campanas Observatory mainly in 1980 May, but with a few additional scans in 1981 May. Two observations were obtained for most of the stars. The first, at a resolution of $1.4 \AA$ (corresponding to the FWHM of the detector of 3.5 pixels) covered the wavelength region from 3700 to $4800 \AA$. These are the observations which were used to obtain the radial velocities. The second, at a lower resolution of $4.0 \AA$, covered the region from 3000 to $5000 \AA$, with a filter strongly suppressing the region longward of 4000 $\AA$. Observations were made by alternating the star between each pair of apertures, separated in declination, so that all the scans were fully sky subtracted. Scans of a tungsten lamp were made to remove pixel-to-pixel variations, while wavelength calibration was achieved using noble gas arcs.

The seeing was significantly better than the width of the aperture $\left(2^{\prime \prime}\right)$ on all nights. However, the spectra were not fluxed. To remove the curvature in the continuum, at least approximately, a third-order polynomial was computed as a fit to the counts within the intervals listed in Table $1 a$; this fit was then used to produce a crudely flattened continuum. Because of the very steep drop-off in continuum signal induced by the long pass cutoff of the filter near the $4700 \AA \mathrm{C}_{2}$ band, the continuum for this feature only was represented by a linear interpolation over the bandpass $4575-4610 \AA$ and $4670-4810$ $\AA$ on either side of the $\mathrm{C}_{2}$ band. The continuum intervals were in all cases chosen to avoid strong atomic or molecular features as much as possible. Of course, especially at wavelengths shortward of $4300 \AA$, it becomes impossible to avoid all features completely in the more metal-rich $\omega$ Cen stars.

Indices were then determined for the bands of $\mathrm{CH}, \mathrm{CN}$, and NH within the spectral range covered on the low- and highresolution scans. These were defined from the sky-subtracted, roughly flattened count rates $\left(c_{i}\right)$ by averaging over appropri- 
TABLE 1

BandPasses for CONTINUUM Flattening

\begin{tabular}{|c|c|c|}
\hline Index & Bandpass $(\AA)$ & Bandpass $(\AA)$ \\
\hline \multicolumn{3}{|c|}{ A. Low Dispersion } \\
\hline $\begin{array}{l}\mathrm{C}_{2} \ldots \ldots \ldots \ldots \ldots \ldots \ldots \ldots \ldots \\
\mathrm{NH}, \mathrm{CN}, \ldots \ldots \ldots \ldots \ldots \ldots\end{array}$ & $\begin{array}{l}4575-4610 \\
3300-3330 \\
3485-3505 \\
3670-3690\end{array}$ & $\begin{array}{l}4760-4810 \\
3890-3910 \\
4025-4050\end{array}$ \\
\hline \multicolumn{3}{|c|}{ B. High Dispersion } \\
\hline $\mathrm{CN}, \mathrm{CH}, \mathrm{H}+\mathrm{K}, \mathrm{Ca} \mathrm{I} \ldots \ldots$ & $\begin{array}{l}3770-3785 \\
3890-3910 \\
3990-4010 \\
4087-4088 \\
4093-4094 \\
4221-4222 \\
4244-4245\end{array}$ & $\begin{array}{l}4335-4336 \\
4345-4346 \\
4365-4366 \\
4396-4397 \\
4402-4403 \\
4448-4449 \\
4477-4478\end{array}$ \\
\hline
\end{tabular}

ate wavelength bandpasses (which process we denote by $A$ ). In general, each index was defined by

$$
I=1-\frac{A\left(c_{i}, \lambda_{1}-\lambda_{2}\right)}{a A\left(c_{i}, \lambda_{3}-\lambda_{4}\right)+b A\left(c_{i}, \lambda_{5}-\lambda_{6}\right)},
$$

where the two wavelength intervals $\lambda_{3}-\lambda_{4}$ and $\lambda_{5}-\lambda_{6}$ represent continuum intervals, and $\lambda_{1}-\lambda_{2}$ is the molecular feature of interest. The continuum weights $a$ and $b$ were chosen so as to minimize the effects of any artificial continuum slope such as could result, for example, from varying amounts of spillover beyond the aperture width due to differential refraction. These weights and the bandpasses are listed for each index in Table 2. The actual wavelength intervals used were obtained from those tabulated by applying a radial velocity correction of $230 \mathrm{~km} \mathrm{~s}^{-1}$ (Webbink 1981) for all the $\omega$ Cen stars.

This approach could not be used for the $4226 \AA$ feature of $\mathrm{Ca}$ I owing to the adjacent $\mathrm{CN}$ and $\mathrm{CH}$ bands. For this feature, a pseudo-equivalent width was measured from the highdispersion scans using a bandpass of 4222-4232 $\AA$. The continuum was defined as above, by fitting a third-order polynomial to selected wavelength intervals listed in Table $1 b$; appropriate radial velocity shifts in the intervals were again made.

A minimum total count of 1000 was always obtained for the continuum bandpass to the blue of the $\mathrm{NH}$ feature; all other wavelength intervals had considerably higher net counts. The

TABLE 2

FEATURES AND WeIGHTS OF THE INTERVALS FOR INDiCES

\begin{tabular}{|c|c|c|c|c|c|}
\hline \multirow[b]{2}{*}{ Feature } & \multirow{2}{*}{$\begin{array}{c}\text { FEATURE BAND } \\
(\AA\end{array}$} & \multirow{2}{*}{$\begin{array}{c}\text { SIDE BAND } \\
(\AA)\end{array}$} & \multirow{2}{*}{$\begin{array}{c}\text { SIDE BAND } \\
(\AA)\end{array}$} & \multicolumn{2}{|c|}{ WEIGHTS } \\
\hline & & & & $a$ & $b$ \\
\hline \multicolumn{6}{|c|}{ A. High Dispersion } \\
\hline $\mathrm{CH}$ & $4290-4320$ & $4350-4370$ & $4235-4270$ & 0.5 & 0.5 \\
\hline $\mathrm{CN}$ & $4160-4210$ & $4115-4130$ & $4235-4270$ & 0.5 & 0.5 \\
\hline uvCN ........... & $3830-3880$ & $3890-3910$ & $3770-3785$ & 0.5 & 0.5 \\
\hline $\mathrm{H}+\mathrm{K} \ldots \ldots \ldots \ldots$ & $3920-3980$ & $3890-3910$ & $3990-4010$ & 0.5 & 0.5 \\
\hline \multicolumn{6}{|c|}{ B. Low Dispersion } \\
\hline NH & $3350-3420$ & $3485-3505$ & $3318-3330$ & 0.33 & 0.67 \\
\hline Low $\mathrm{CN} \ldots \ldots$ & $3820-3870$ & $3900-3910$ & $3650-3700$ & 0.67 & 0.33 \\
\hline $\mathrm{C}_{2} \ldots \ldots \ldots \ldots \ldots$ & $4660-4720$ & $4760-4800$ & $4575-4610$ & 0.5 & 0.5 \\
\hline
\end{tabular}

principal uncertainty is not in the count rates within any bandpass themselves, but rather in the measurement of indices in the absence of fluxed spectra. This resulting uncertainty in the tabulated indices is believed to be $\pm 4 \%$; the pseudoequivalent width of the $4226 \AA$ feature of $\mathrm{Ca}$ I has a larger uncertainty of $\pm 10 \%$. The two independent measurements of the $3883 \AA \mathrm{CN}$ band, one from the low and one from the high-dispersion scans, indicate the good accuracy of these measurements. Duplicate scans of five stars also support the claimed high accuracy of these molecular indices.

The star RGO 300 is a radial velocity member of $\omega$ Cen with $\mathrm{TiO}$ and $\mathrm{ZrO}$ bands classified S2 by Lloyd-Evans (1983). Our three spectra reveal the presence of a hot companion which is contributing significantly to the total light at wavelengths of $4000 \AA$ and below. The higher Balmer lines and a strong uv continuum are seen, and the $\mathrm{Ca}$ II $-\mathrm{H}_{\epsilon}$ blend is stronger than the $3933 \AA \mathrm{Ca}$ II line. Consequently, we do not consider this star further in the present paper and its indices are omitted from all the figures.

The measured molecular indices and atomic line strengths for the 72 program stars are given in Table 3.

\section{THE CORRELATIONS OF THE MOLECULAR BAND INDICES AND ATOMIC LINE STRENGTHS}

The displacement of a star in the $M_{V}, V-K$ diagram with respect to the M92 fiducial giant branch at the same $m_{\text {bol }}$ was used by PFCAM to define the parameter $R(V-K)$; observationally, $R(V-K)$ is equal to 0.71 for a star with the metallicity of an M71 star. We use this parameter to separate the stars in metallicity. In order to have metallicity values available for subsequent use we adopt $[M / \mathrm{H}]=-2.1$ dex for $\mathrm{M} 92$ $[R(V-K)=0.0]$ and $[M / \mathrm{H}]=-0.8$ dex for $\mathrm{M} 71$ $[R(V-K)=0.71]$ (Cohen 1983; Bell and Gustafsson 1983). For $\mathrm{M} 13, R(V-K)=0.3$, and we adopt $[M / \mathrm{H}]=-1.5 \mathrm{dex}$ (Cohen 1978; Bell and Dickens 1980). This separation in metallicity given by the $R(V-K)$ parameter is supported by the high-dispersion analyses of several $\omega$ Cen members by Cohen (1981), Mallia and Pagel (1981), and Gratton (1982), as well as by the trends in line strength shown by the atomic features as displayed subsequently here.

The figures are separated into three panels covering the three metallicity ranges: the most metal-poor $\omega$ Cen stars $[R(V-K)<0.20]$, the intermediate metallicity giants $[0.20 \leq R(V-K) \leq 0.40]$, and the high metallicity giants $[R(V-K)>0.40]$. The abundances which correspond to these three groups are taken to be $-2.0,-1.5$, and -1.0 dex respectively, based on the discussion above. Because atomic and molecular line strengths depend on both $T_{\text {eff }}$ and metallicity (as well as surface gravity), such a complex display of the data becomes necessary.

We first consider the $\mathrm{C}_{2}$ bands. Only four stars have a $\mathrm{C}_{2}$ index greater than $10 \%$; the $\mathrm{C}_{2}$ indices of the remaining stars fluctuate about zero. RGO 55 and RGO 70 are well known $\mathrm{CH}$ stars (Harding 1972; Dickens 1972), while RGO 577 was found by this survey to have strong $\mathrm{C}_{2}$ bands; unpublished observations by Zinn and Norris also had independently found this star to be a carbon star. RGO 577 is a radial velocity member of the globular cluster, with $M_{\text {bol }}$ (from PFCAM) of $-1.45 \mathrm{mag}$, well below that of the tip of the giant branch. RGO 279 is also a carbon star, with weaker $\mathrm{C}_{2}$ bands and a smaller value of $\mathrm{C} / \mathrm{O}$. A fifth carbon star was found by Bond (1975), near the cluster center. Models for these carbon-rich objects have not yet been computed, and the observations are 
TABLE 3

INDICES OF $\omega$ CENTAURI STARS

\begin{tabular}{|c|c|c|c|c|c|c|c|c|c|c|c|}
\hline $\begin{array}{r}\text { RGO } \\
(\#)\end{array}$ & $\begin{array}{r}(\mathrm{V}-\mathrm{K})_{o} \\
(\mathrm{mag})\end{array}$ & $\begin{array}{c}\mathrm{CO} \\
(\mathrm{mag})\end{array}$ & $\begin{array}{l}\mathrm{CH} \\
(\%)\end{array}$ & $\begin{array}{l}\mathrm{CN} \\
(\%)\end{array}$ & $\begin{array}{l}\text { UVCN } \\
(\%)\end{array}$ & $\begin{array}{r}\text { LOCN } \\
(\%)\end{array}$ & $\begin{array}{l}\mathrm{NH} \\
(\%)\end{array}$ & $\begin{array}{l}\mathrm{C}_{2} \\
(\%)\end{array}$ & $\begin{array}{c}\mathrm{H}+\mathrm{K} \\
(\%)\end{array}$ & $\begin{array}{l}\mathrm{CaI} \\
(\mathrm{A})\end{array}$ & Group \\
\hline \multicolumn{12}{|c|}{ Low Metallicity Group } \\
\hline 40 & 3.14 & 0.095 & 0.228 & 0.085 & 0.328 & 0.345 & 0.175 & 0.028 & 0.412 & 1.88 & L \\
\hline 46 & 3.41 & 0.040 & 0.210 & 0.039 & 0.140 & 0.188 & 0.097 & -0.037 & 0.445 & 2.78 & $\mathrm{U}$ \\
\hline 48 & 3.40 & 0.040 & 0.226 & 0.057 & 0.176 & 0.200 & -0.040 & -0.004 & 0.394 & 1.69 & $\mathrm{U}$ \\
\hline 49 & 3.34 & 0.060 & 0.229 & 0.066 & 0.102 & 0.145 & 0.147 & 0.025 & 0.375 & 1.93 & $\mathrm{~L}$ \\
\hline 55 & 3.49 & 0.060 & 0.150 & 0.484 & 0.735 & 0.786 & 0.067 & 0.663 & 0.189 & -0.78 & C \\
\hline 58 & 3.14 & 0.020 & 0.203 & 0.058 & 0.214 & 0.222 & 0.257 & -0.040 & 0.437 & 1.71 & $\mathrm{U}$ \\
\hline 61 & 3.48 & 0.050 & 0.216 & 0.047 & 0.192 & 0.221 & 0.197 & -0.068 & 0.369 & 2.55 & $\mathrm{U}$ \\
\hline 62 & 3.43 & 0.070 & 0.193 & 0.079 & 0.192 & 0.245 & -0.032 & -0.015 & 0.411 & 2.24 & $\mathrm{~L}$ \\
\hline 70 & 3.42 & 0.150 & 0.219 & 0.365 & 0.690 & 0.699 & 0.164 & 0.309 & 0.335 & 1.56 & $\mathrm{C}$ \\
\hline 74 & 3.03 & 0.035 & 0.195 & 0.044 & 0.221 & 0.202 & 0.253 & 0.034 & 0.432 & 1.50 & $\mathrm{U}$ \\
\hline 102 & 3.17 & 0.015 & 0.187 & 0.058 & 0.164 & 0.216 & 0.192 & -0.029 & 0.414 & 1.61 & $\mathrm{U}$ \\
\hline 124 & 3.09 & 0.060 & 0.195 & 0.028 & 0.189 & 0.214 & 0.177 & -0.062 & 0.451 & 1.79 & $\mathrm{~L}$ \\
\hline 159 & 2.97 & 0.040 & 0.222 & 0.033 & 0.147 & 0.141 & 0.170 & -0.043 & 0.414 & 1.65 & $\mathrm{U}$ \\
\hline 180 & 2.76 & 0.035 & 0.189 & 0.020 & 0.007 & 0.070 & 0.155 & -0.035 & 0.362 & 1.44 & $\mathrm{U}$ \\
\hline 213 & 2.67 & 0.010 & 0.218 & 0.000 & 0.047 & 0.080 & 0.150 & -0.074 & 0.379 & 0.49 & $\mathrm{U}$ \\
\hline 234 & 2.70 & 0.010 & 0.171 & 0.021 & 0.041 & 0.088 & 0.201 & -0.036 & 0.362 & 1.45 & $\mathrm{U}$ \\
\hline 256 & 2.80 & 0.001 & 0.203 & 0.036 & 0.262 & 0.256 & 0.275 & -0.043 & 0.422 & 1.19 & $\mathrm{U}$ \\
\hline 269 & 2.79 & 0.025 & 0.208 & 0.018 & 0.072 & 0.129 & 0.107 & -0.104 & 0.451 & 1.23 & $\mathrm{U}$ \\
\hline 279 & 2.81 & 0.105 & 0.245 & 0.236 & 0.595 & 0.587 & 0.165 & 0.135 & 0.343 & 1.07 & C \\
\hline 364 & 2.57 & 0.001 & 0.209 & 0.020 & 0.141 & $\ldots$ & $\ldots$ & $\ldots$ & 0.373 & 0.52 & $\mathrm{U}$ \\
\hline 402 & 2.53 & 0.005 & 0.123 & 0.043 & 0.147 & 0.113 & 0.295 & -0.018 & 0.351 & 0.00 & $\mathrm{U}$ \\
\hline 415 & 2.50 & 0.015 & 0.237 & 0.022 & 0.236 & $\ldots$ & $\ldots$ & $\ldots$ & 0.355 & 0.96 & $\mathrm{U}$ \\
\hline 461 & 2.61 & 0.055 & 0.240 & 0.069 & 0.415 & $\ldots$ & $\ldots$ & $\ldots$ & 0.445 & 1.67 & $\mathrm{~L}$ \\
\hline 462 & 2.40 & $\ldots$ & 0.122 & 0.026 & 0.084 & $\ldots$ & $\ldots$ & $\ldots$ & 0.354 & 0.80 & Ù \\
\hline 483 & 2.56 & 0.015 & 0.175 & -0.010 & 0.088 & $\ldots$ & $\ldots$ & $\ldots$ & 0.357 & 0.68 & $\mathrm{U}$ \\
\hline 509 & 2.53 & $\ldots$ & 0.174 & 0.028 & 0.168 & $\ldots$ & $\ldots$ & $\ldots$ & 0.374 & 1.40 & $\mathrm{U}$ \\
\hline G318 & 2.40 & $\ldots$ & 0.259 & 0.053 & 0.342 & $\ldots$ & $\ldots$ & $\ldots$ & 0.377 & 0.85 & $\mathrm{U}$ \\
\hline 5941 & 2.16 & $\ldots$ & 0.149 & 0.020 & -0.010 & $\ldots$ & $\ldots$ & $\ldots$ & 0.271 & 0.48 & $\mathrm{U}$ \\
\hline 6113 & 2.25 & $\ldots$ & 0.201 & 0.044 & 0.246 & $\ldots$ & $\ldots$ & $\ldots$ & 0.377 & 0.93 & $\mathrm{U}$ \\
\hline \multicolumn{12}{|c|}{ Intermediate Metallicity Group } \\
\hline 43 & 3.60 & 0.140 & 0.228 & 0.145 & 0.284 & 0.334 & 0.166 & 0.025 & 0.446 & 2.62 & L \\
\hline 53 & 3.62 & 0.105 & 0.189 & 0.079 & 0.197 & 0.252 & 0.207 & 0.071 & 0.431 & 2.15 & L \\
\hline 56 & 3.53 & 0.060 & 0.225 & 0.144 & 0.279 & 0.374 & 0.236 & 0.003 & 0.457 & 2.74 & $\mathrm{U}$ \\
\hline 84 & 3.68 & 0.180 & 0.221 & 0.076 & 0.275 & 0.326 & 0.253 & -0.002 & 0.511 & 4.02 & $\mathrm{~L}$ \\
\hline 90 & 3.46 & 0.045 & 0.230 & 0.077 & 0.215 & 0.310 & 0.317 & -0.034 & 0.466 & 2.88 & $\mathrm{U}$ \\
\hline 91 & 3.14 & 0.015 & 0.206 & 0.031 & 0.143 & 0.170 & 0.144 & 0.002 & 0.419 & 1.43 & $\mathrm{U}$ \\
\hline 96 & 3.26 & 0.050 & 0.219 & 0.038 & 0.125 & 0.171 & 0.068 & -0.019 & 0.432 & 2.22 & $\mathrm{U}$ \\
\hline 132 & 3.72 & 0.155 & 0.229 & 0.107 & 0.259 & 0.304 & 0.140 & 0.021 & 0.466 & 3.24 & $\mathrm{~L}$ \\
\hline 139 & 3.17 & 0.135 & 0.235 & 0.202 & 0.510 & 0.539 & 0.282 & -0.029 & 0.440 & 1.92 & L \\
\hline 150 & 3.53 & 0.070 & 0.215 & 0.209 & 0.363 & 0.438 & 0.286 & 0.050 & 0.574 & 3.82 & w \\
\hline 155 & 3.11 & 0.075 & 0.235 & 0.055 & 0.248 & 0.266 & 0.184 & 0.033 & 0.428 & 2.42 & $\mathrm{~L}$ \\
\hline 161 & 3.03 & 0.070 & 0.229 & 0.027 & 0.255 & 0.265 & 0.199 & -0.002 & 0.406 & 2.10 & L \\
\hline 171 & 3.28 & 0.145 & 0.234 & 0.081 & 0.287 & 0.338 & 0.214 & -0.007 & 0.487 & 3.05 & $\mathrm{~L}$ \\
\hline 253 & 2.93 & 0.005 & 0.223 & 0.229 & 0.561 & 0.582 & 0.312 & -0.047 & 0.477 & 2.16 & $\mathrm{U}$ \\
\hline 272 & 2.96 & 0.085 & 0.235 & 0.100 & 0.402 & 0.426 & 0.192 & -0.053 & 0.461 & 1.79 & $\mathrm{~L}$ \\
\hline 287 & 3.01 & 0.150 & 0.257 & 0.119 & 0.411 & 0.434 & 0.194 & 0.016 & 0.485 & 2.50 & L \\
\hline 297 & 2.85 & 0.085 & 0.246 & 0.048 & 0.313 & 0.350 & 0.241 & -0.025 & 0.440 & 2.12 & L \\
\hline 312 & 2.90 & 0.065 & 0.237 & 0.041 & 0.308 & $\ldots$ & $\ldots$ & $\ldots$ & 0.440 & 1.35 & L \\
\hline 320 & 3.80 & 0.300 & 0.192 & 0.086 & 0.136 & 0.209 & 0.117 & -0.010 & 0.410 & 4.58 & L \\
\hline 394 & 2.92 & 0.105 & 0.248 & 0.170 & 0.483 & 0.505 & 0.298 & 0.004 & 0.509 & 2.49 & L \\
\hline 464 & 2.65 & 0.070 & 0.249 & 0.024 & 0.299 & 0.245 & 0.194 & 0.057 & 0.374 & 1.34 & L \\
\hline 465 & 2.87 & 0.050 & 0.246 & 0.194 & 0.493 & 0.531 & 0.352 & -0.048 & 0.495 & 2.35 & W \\
\hline 472 & 2.84 & 0.135 & 0.260 & 0.079 & 0.374 & $\ldots$ & $\ldots$ & $\ldots$ & 0.510 & 2.44 & L \\
\hline 480 & 2.80 & 0.060 & 0.256 & 0.318 & 0.626 & 0.672 & 0.279 & 0.021 & 0.572 & 2.77 & W \\
\hline 505 & 2.87 & 0.045 & 0.244 & 0.272 & 0.547 & 0.647 & 0.328 & -0.017 & 0.560 & 2.95 & W \\
\hline 537 & 2.74 & 0.100 & 0.253 & 0.040 & 0.390 & $\ldots$ & $\cdots$ & $\ldots$ & 0.460 & 1.19 & L \\
\hline 557 & 2.82 & 0.055 & 0.246 & 0.272 & 0.619 & 0.694 & 0.386 & 0.001 & 0.518 & 2.34 & $\mathrm{~W}$ \\
\hline 577 & 2.71 & 0.120 & 0.134 & 0.262 & 0.606 & 0.665 & 0.037 & 0.467 & 0.243 & -0.65 & C \\
\hline
\end{tabular}


TABLE 3-Continued

\begin{tabular}{|c|c|c|c|c|c|c|c|c|c|c|c|}
\hline $\begin{array}{r}\text { RGO } \\
(\#)\end{array}$ & $\begin{array}{r}(\mathrm{V}-\mathrm{K})_{o} \\
(\mathrm{mag})\end{array}$ & $\begin{array}{c}\mathrm{CO} \\
(\mathrm{mag})\end{array}$ & $\begin{array}{l}\mathrm{CH} \\
(\%)\end{array}$ & $\begin{array}{l}\text { CN } \\
(\%)\end{array}$ & $\begin{array}{c}\text { UVCN } \\
(\%)\end{array}$ & $\begin{array}{r}\text { LOCN } \\
(\%)\end{array}$ & $\begin{array}{l}\text { NH } \\
(\%)\end{array}$ & $\begin{array}{l}\mathrm{C}_{2} \\
(\%)\end{array}$ & $\begin{array}{c}\mathrm{H}+\mathrm{K} \\
(\%)\end{array}$ & $\begin{array}{l}\mathrm{CaI} \\
(\mathrm{A})\end{array}$ & Group \\
\hline \multicolumn{12}{|c|}{ High Metallicity Group } \\
\hline 162 & 3.52 & 0.080 & 0.228 & 0.267 & 0.459 & 0.529 & 0.251 & 0.002 & 0.541 & 3.22 & W \\
\hline 179 & 3.76 & 0.195 & 0.217 & 0.079 & 0.276 & 0.313 & 0.233 & 0.022 & 0.532 & 4.73 & L \\
\hline 201 & 3.88 & 0.230 & 0.233 & 0.127 & 0.241 & 0.322 & 0.125 & 0.033 & 0.508 & 4.69 & $\mathrm{~L}$ \\
\hline 219 & 3.43 & 0.180 & 0.233 & 0.096 & 0.309 & 0.337 & 0.159 & -0.024 & 0.493 & 3.49 & L \\
\hline 231 & 3.45 & 0.080 & 0.241 & 0.183 & 0.239 & 0.415 & 0.235 & -0.018 & 0.522 & 4.35 & W \\
\hline 248 & 3.72 & 0.100 & 0.232 & 0.183 & 0.377 & 0.395 & 0.244 & 0.037 & 0.554 & 4.64 & W \\
\hline 270 & 3.26 & 0.200 & 0.225 & 0.132 & 0.343 & 0.434 & 0.211 & -0.046 & 0.489 & 3.36 & L \\
\hline 300 & 3.62 & 0.155 & 0.163 & 0.103 & 0.101 & 0.197 & 0.050 & 0.039 & 0.280 & 4.74 & . \\
\hline 357 & 3.47 & 0.130 & 0.258 & 0.215 & 0.377 & 0.441 & 0.247 & 0.017 & 0.555 & 5.04 & W \\
\hline 421 & 3.02 & 0.060 & $\ldots$ & $\ldots$ & $\ldots$ & 0.611 & 0.279 & 0.005 & $\ldots$ & $\ldots$ & W \\
\hline 451 & 3.31 & 0.110 & 0.204 & 0.183 & 0.352 & 0.428 & 0.185 & -0.002 & 0.492 & 4.09 & W \\
\hline 371 & 3.45 & 0.105 & 0.218 & 0.179 & 0.317 & 0.468 & 0.222 & 0.049 & 0.561 & 4.28 & W \\
\hline 425 & 3.99 & 0.155 & 0.210 & 0.148 & 0.213 & 0.334 & 0.131 & 0.025 & 0.578 & 5.56 & W \\
\hline 447 & 3.94 & 0.110 & 0.205 & 0.145 & 0.220 & 0.328 & 0.227 & -0.009 & 0.518 & 5.94 & $\mathbf{w}$ \\
\hline 513 & 3.99 & 0.145 & 0.205 & 0.142 & 0.301 & 0.351 & 0.377 & -0.016 & 0.587 & 5.96 & W \\
\hline
\end{tabular}

not discussed in detail. However, we note that both RGO 279 and 577 have very strong $\mathrm{CN}$ bands, similar to those seen in RGO 55 and 70. The synthetic spectra of Bell and Dickens (1974) show that strong $\mathrm{CN}$ occurs in $\mathrm{CH}$ stars even if the nitrogen abundance is not enhanced. Not all stars with very strong $\mathrm{CN}$ are $\mathrm{CH}$ stars, however, and RGO 253 has very strong $\mathrm{CN}$ bands and negligible $\mathrm{C}_{2}$ and $\mathrm{CO}$.

The $\mathrm{CO}$ indices of all the stars have been measured by PFCAM. In their Figure 5, stars classified into three groups. In the first, denoted in the final column of Table 3 as " $L$ " (and plotted as open squares), PFCAM argued that the CO index was abnormally large for the metallicity and $T_{\text {eff }}$ of the star, Such stars are on the more vertical sequence of Figure 5 of PFCAM. There is also a group of stars with weaker CO indices for their metallicity and temperature, denoted " $\mathrm{W}$ " in the last column of Table 3 and plotted as filled circles. In the lowest metallicity or the hottest stars, the $\mathrm{CO}$ band is so weak that no separation of the two groups can be achieved. Such stars, plus four of the hotter stars which have no $\mathrm{CO}$ measurements, are denoted " $U$ " in the last column of the table and are always plotted as open circles. The four carbon stars are denoted by $\mathrm{C}$ in the tables and plotted as stars. It is clear that the $\mathrm{C} / \mathrm{O}$ ratio in the $\mathrm{CH}$ stars varies from one another. For example, RGO 55 and 70 have the same $V-K$ color (and therefore the same temperature), but RGO 70 has much stronger $\mathrm{CO}$ and weaker $\mathrm{C}_{2}$ and therefore a much lower $\mathrm{C} / \mathrm{O}$ ratio.

The $\mathrm{CO}$ band indices are plotted as a function of reddening corrected $(V-K)_{0}$ color in Figure $1 a-c$; the symbols are as defined above. These bands show a great range in strength even when the stars are separated into the different abundance groups. In the lowest abundance group, the range is largely caused by the presence of the $\mathrm{CH}$ stars RGO 70 and 279, both of which have very strong $\mathrm{CO}$ for this group. However, the two normal giants RGO 40 and 461 also have larger CO indices than the other stars of the same $V-K$ color in the group. PFCAM also drew attention to the fact that many of the $\omega$ Cen giants have very strong $\mathrm{CO}$ compared with stars in other clusters. Examples of these stars are RGO 170, 201, 219, and 270 . The possibility that these stars are simply extremely metal rich will be examined later. In all the clusters observed by Frogel, Persson, and Cohen (1983), stars with CO bands of this strength at $V-K$ color near $3.5 \mathrm{mag}$ are seen only in NGC 5927. All the 47 Tuc stars have weaker CO.

In Figure $2 a-c$ we plot the $\mathrm{CH}$ index as a function of the reddening corrected $(V-K)_{0}$ color for the 72 stars. The symbols are as defined above. Figure 3 is completely analogous for the $4200 \AA \mathrm{CN}$ band, while the mean of two independent measurements of the $3883 \AA \mathrm{CN}$ band is shown in Figure 4 . The behavior of the NH index in $\omega$ Cen giants is displayed in Figure 5. Figure $6 a-c$ presents the $\mathrm{H}+\mathrm{K}$ indices as a function of $T_{\text {eff }}$ for the three metallicity bins, while Figure $7 a-c$ does so for the pseudo-equivalent width of the $4226 \AA \mathrm{Ca}$ I feature.

The $\mathrm{CH}$ indices plotted in Figure 2 show no separation between the $\mathrm{CO}$-strong and $\mathrm{CO}$-weak groups of $\omega$ Cen giants. Furthermore, there is at best only a slight increase in the mean $\mathrm{CH}$ index at a fixed $T_{\text {eff }}$ as the mean metallicity increases. Excluding the $\mathrm{CH}$ stars, while there may be a range in the strength of the $\mathrm{CH}$ band among the $\omega$ Cen giants, it is not a large one.

Norris and Bessell (1975) and Dickens and Bell (1976) showed that the $\mathrm{CN}$ bands have a truly impressive range of strengths among $\omega$ Cen stars. Figures 3 and 4 also show the great range in $\mathrm{CN}$ among stars of the same $T_{\text {eff }}$ and metallicity group. There are many stars which do not show detectable $\mathrm{C}_{2}$ bands and yet have $\mathrm{CN}$ bands as strong as those of the carbon stars. It is also clear from Figures 3 and 4 that within each metallicity group, the $\mathrm{CO}$ strong stars have generally weaker $\mathrm{CN}$ than their counterparts at the same $T_{\text {eff }}$ among the $\mathrm{CO}$ weak stars. The $\mathrm{CN}$ band strengths reached by some of the $\omega$ Cen giants, even those from only the two lowest metallicity groups, are truly phenomenal. They exceed the strength of the $\mathrm{CN}$ bands in even the $\mathrm{CN}$-strong 47 Tuc stars, a few of which were observed and reduced in the same manner as the $\omega$ Cen giants.

The NH bands also show a considerable variation in strength among the $\omega$ Cen stars. In general, the stronger $\mathrm{NH}$ bands within each panel of Figure 5 are found among the group of $\mathrm{CO}$ weak stars.

To summarize, for stars of a given $T_{\text {eff }}$ and heavy element metallicity, (1) CN strength is in general stronger in the $\mathrm{CO}$ weak stars; (2) NH strength is stronger in the $\mathrm{CO}$ weak stars; (3) $\mathrm{CH}$ does not vary much. 

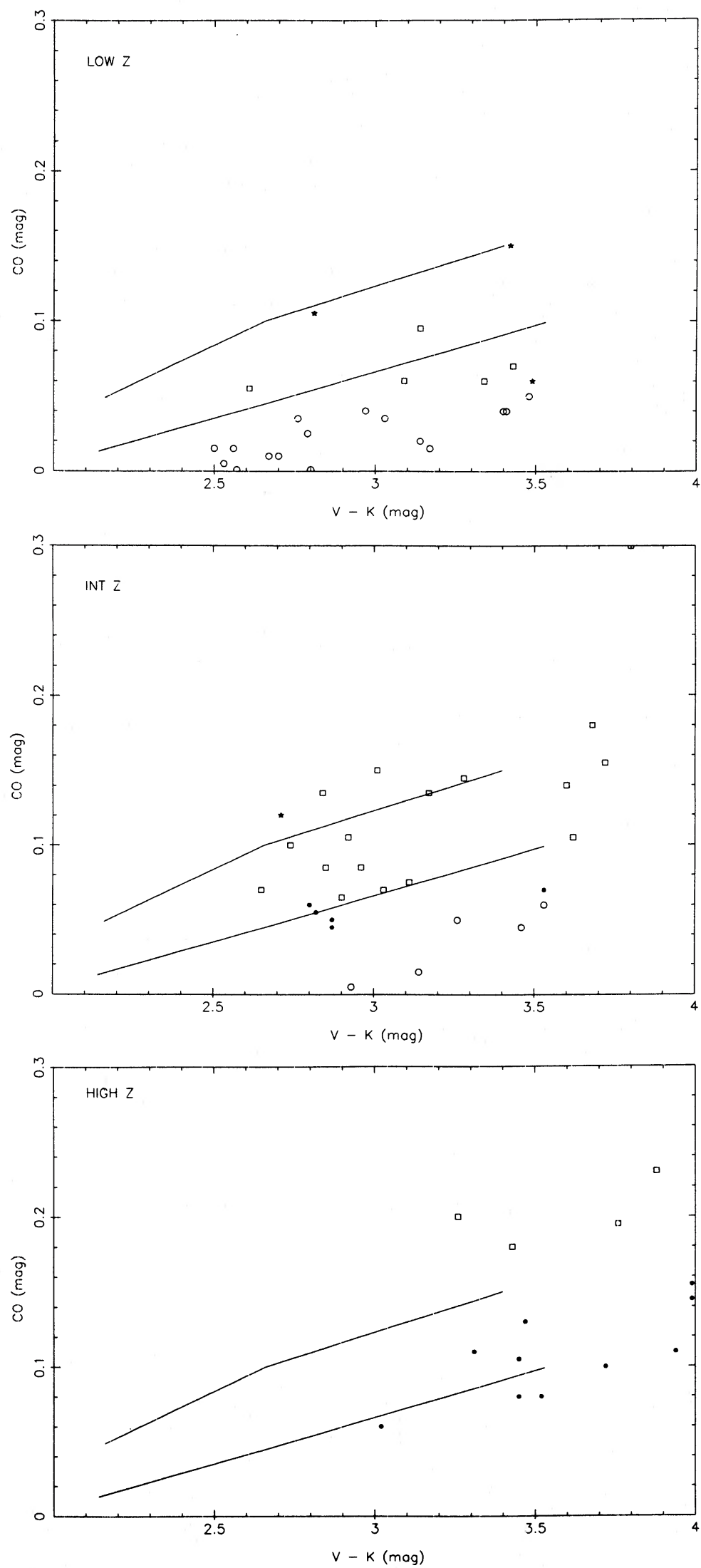

FIG. 1.-The indices of the strength of the $2.4 \mu \mathrm{m} \mathrm{CO}$ band from PFCAM for $\omega$ Cen stars are shown as a function of unreddened $V-K$ color. The three panels display the low, intermediate, and high metallicity groups of the sample of $\omega$ Cen giants. The four carbon stars are indicated by asterisks. The filled circles denote the $\mathrm{CO}$ weak-CN strong stars, while the open squares are the $\mathrm{CO}$ strong- $\mathrm{CN}$ weak stars. The open circles are the hotter stars or those of very low metallicity with such weak $\mathrm{CO}$ bands that no separation of the two groups is possible. The two solid lines connect the CO indices predicted by the synthetic spectra computed from model atmospheres at $T_{\text {eff }}=4000,4500$, and $5000 \mathrm{~K}$ and of metallicity of $1 / 10$ and $1 / 100$ solar. The surface gravities of the model atmospheres at each $T_{\text {eff }}$ correspond to globular cluster giants of the appropriate metallicity. 

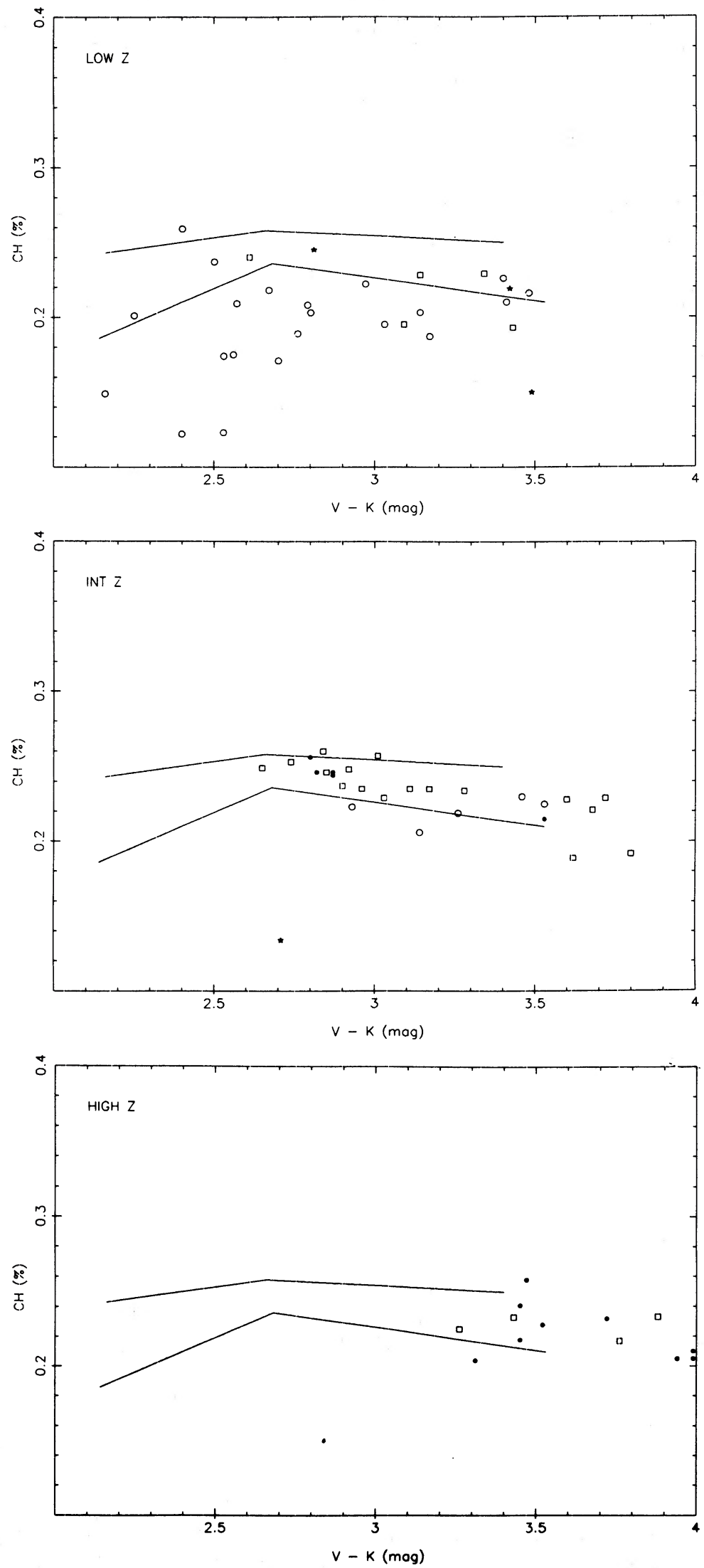

Fig. 2.-The indices for the strenth of the $4300 \AA \mathrm{CH}$ band for $\omega$ Cen stars are plotted as a function of unreddened $V-K$ color. The symbols are as in Fig. 1 . The solid lines represent the $\mathrm{CH}$ indices predicted from model atmospheres appropriate to globular cluster giants with metallicities of 1/10 and 1/100 solar as in Fig. 1 . 

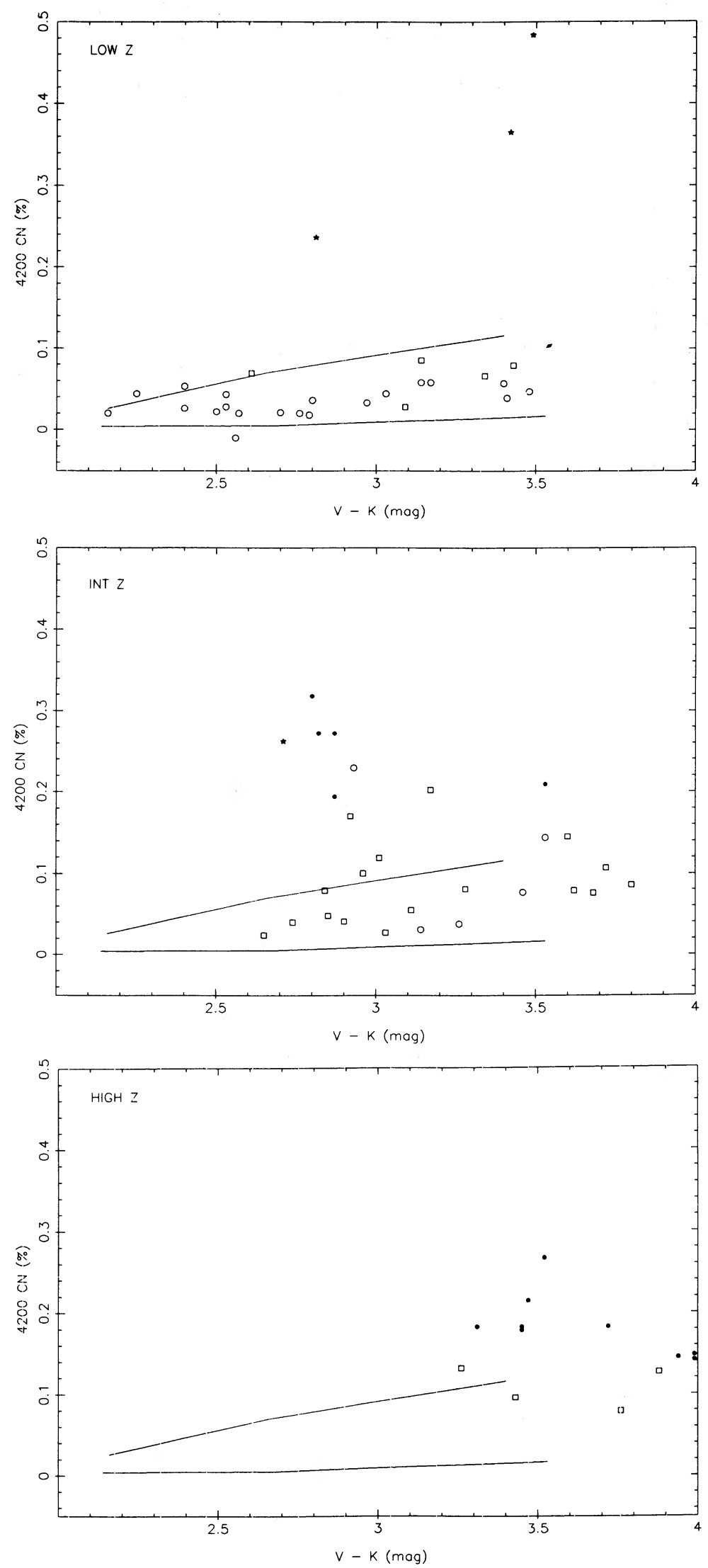

Fig. 3. - The indices of the strength of the $4200 \AA \mathrm{CN}$ band in a sample of $\omega$ Cen stars are displayed as a function of unreddened $V-K$ color. The symbols are as in Fig. 1. The solid lines represent the $\mathrm{CN}$ indices predicted from model atmospheres appropriate to globular cluster giants with metallicities of $1 / 10$ and $1 / 100$ solar. 

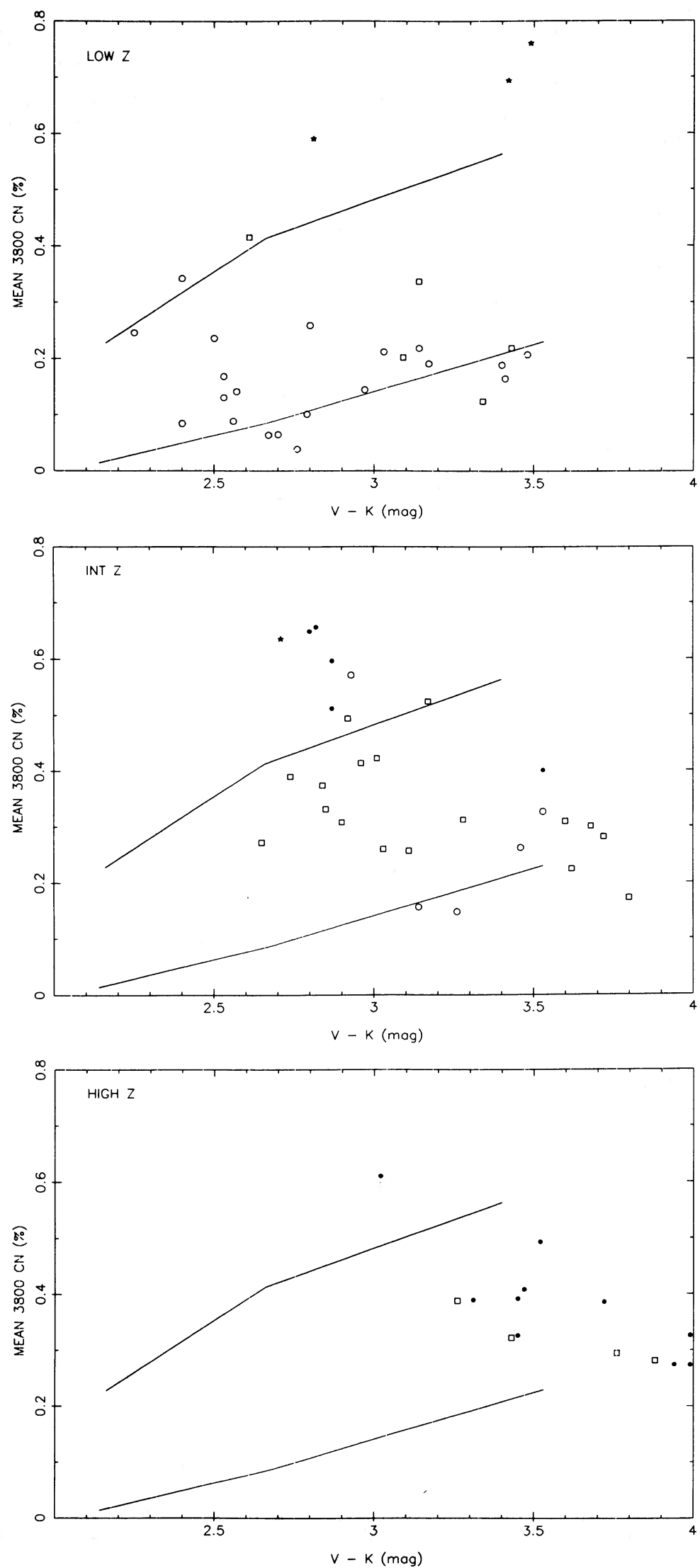

Fig. 4. - The mean of two independent measurements of the strength of the $3883 \AA \mathrm{CN}$ band in $\omega$ Cen stars is plotted as a function of unreddened $V-K$ color. The symbols are as in Fig. 1. The solid lines display the mean of the two predicted $\mathrm{CN}$ indices from model atmospheres appropriate for globular cluster giants with metallicities of $1 / 10$ and $1 / 100$ solar. 

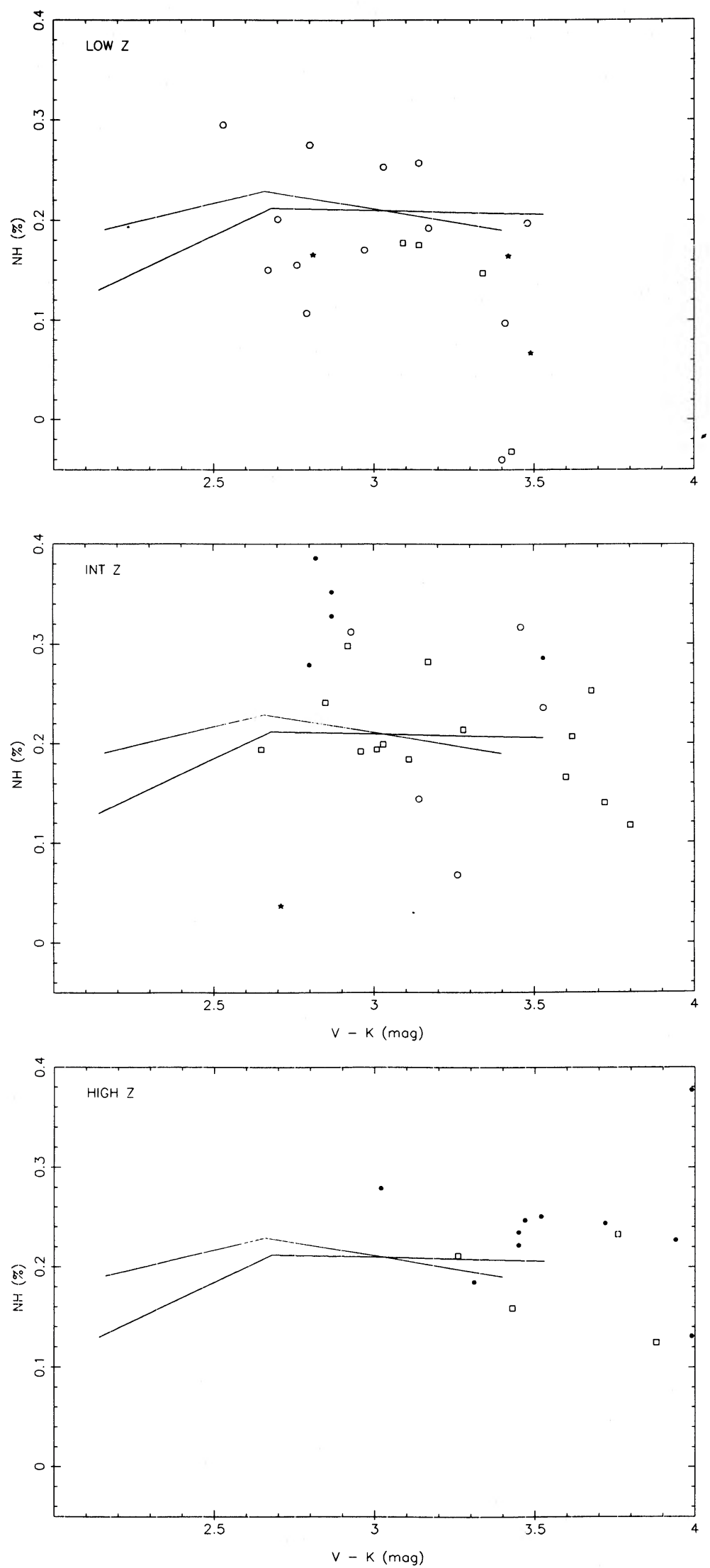

Fig. 5.-The indices of the strength of the $3360 \AA \mathrm{NH}$ band is a sample of $\omega$ Cen giants is shown as a function of unreddened $V-K$ color. The symbols are as in Fig. 1. The solid lines represent the NH indices predicted from model atmospheres appropriate for globular cluster giants with metallicities of $1 / 10$ and $1 / 100$ solar. 

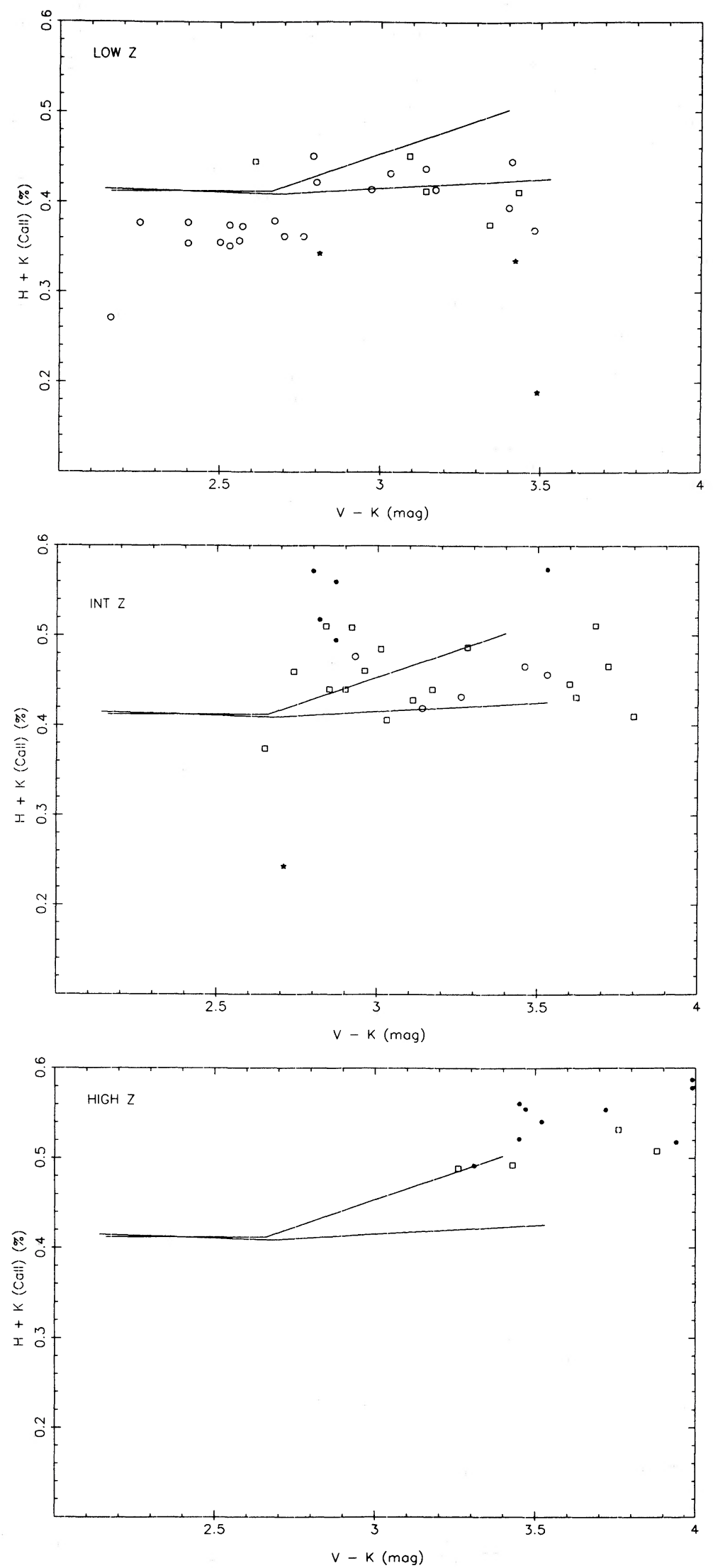

FiG. 6. - The $\mathrm{H}+\mathrm{K}$ indices of the strength of the Ca II resonance lines are shown as a function of unreddened $V-K$ color for a sample of $\omega$ Cen stars. The symbols are identical to those in Fig. 1. The solid lines represent the predicted $\mathrm{H}+\mathrm{K}$ indices from model atmospheres appropriate to globular cluster red giants of $1 / 10$ and $1 / 100$ solar metallicity. 

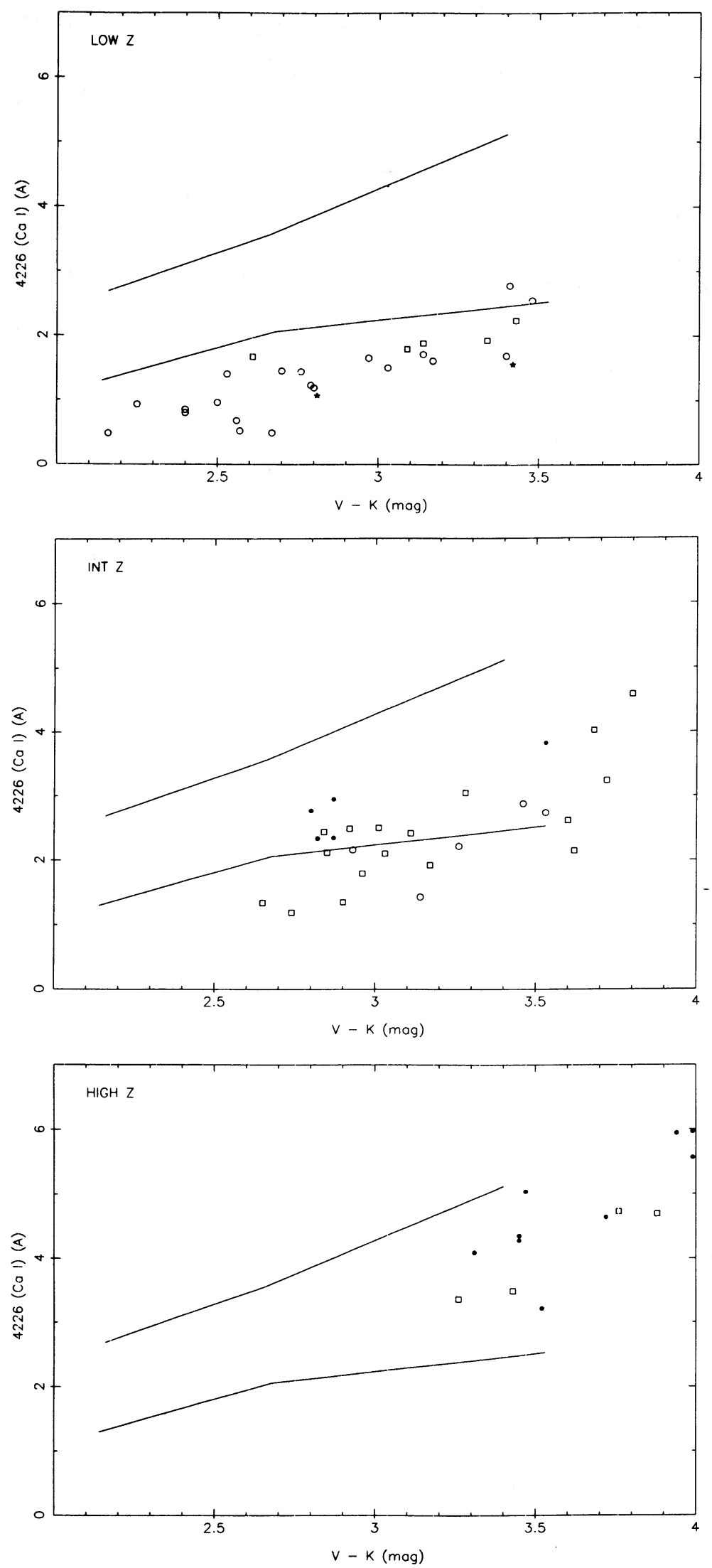

FIG. 7.-The pseudo-equivalent width of the $4226 \AA \mathrm{Ca}$ I feature is displayed as function of unreddened $V-K$ color for a sample of $\omega$ Cen stars. The symbols are as in Fig. 1. The solid lines represent the predicted strength of the $\mathrm{Ca}$ I feature calculated from model atmospheres appropriate to globular cluster giants of $1 / 10$ and $1 / 100$ solar metallicity. 


\section{CALIBRATION OF THE INDICES}

It is known that in several of the globular clusters, including M92 and NGC 6397, the most luminous stars are depleted in carbon. It is commonly believed that this depletion results from the mixing of nuclear processed material from the stellar interior to the surface. However, in addition to material depleted in carbon, it is also possible for material depleted in oxygen to be mixed to the surface as well. The relative abundances of carbon, nitrogen, and oxygen are such that minor depletions result in significant enhancement of nitrogen. The question then arises as to whether it is possible for values of $\mathrm{C}$, $\mathrm{N}$, and $\mathrm{O}$ to be chosen such that the observed $\mathrm{CH}, \mathrm{CO}$, and NH band strengths in the $\omega$ Cen giants can be reproduced simultaneously. Once complicating factor (which we ignore here) is whether the difference in isotope ratios from star to star is significant.

\section{a) Synthetic Spectra}

Bell and Gustafsson (1983) have computed synthetic spectra for models with $T_{\text {eff }}$, surface gravity, and metallicity in the range covered by globular cluster stars. Some additional calculations were made for the present paper, using their methods and programs. Line absorption from the relevant molecular bands of $\mathrm{CH}, \mathrm{CN}, \mathrm{NH}$, and $\mathrm{CO}$ as well as atomic lines are included in these spectral syntheses, details of the sources of these data being given by Bell and Gustafsson (1978).

The theoretical molecular band indices were calculated for $T_{\text {eff }}=4000,4500$, and $5000 \mathrm{~K}$ for $Z=1 / 3,1 / 10$, and $1 / 100 Z_{0}$ at surface gravities which for each $T_{\text {eff }}$ corresponds to those of globular cluster giants. A number of additional models were computed for lower gravities in order to study the abundances of AGB stars, and some further models were computed using nitrogen abundances enhanced by $1 \mathrm{dex}$. The logarithmic $\mathrm{C}$, $\mathrm{N}$, and $\mathrm{O}$ abundances accepted as standard are 8.62, 8.00, and 8.86 , on the scale with $\log N(\mathrm{H})=12.0$. Calculations have been carried out using these values and a variety of other values. In particular, we have carried out the calculations for reduced $\mathrm{C}$ and $\mathrm{O}$ abundances, allowing the $\mathrm{N}$ abundance to increase as a result of assumed $\mathrm{CN}$ and $\mathrm{ON}$ processing. Clearly, the conclusions of the previous section suggest that both $\mathrm{CN}$ and $\mathrm{ON}$ processed material have been brought to the stellar surface. Synthetic spectra in the $2 \mu \mathrm{m}$ to $2.5 \mu \mathrm{m}$ region were used to interpret the PFCAM CO data. Similar calculations were used by Bell, Dickens, and Gustafsson (1979) to check carbon abundances in the M92 stars. Infrared synthetic spectra were computed for the models with $[N / \mathrm{A}]=1.0 \mathrm{dex}$, to check the effect of $\mathrm{CN}$ absorption on the infrared $\mathrm{CO}$ band photometry.

The synthetic spectra covering the ultraviolet and visible spectral regions were flattened in a manner identical to the treatment of the observations, and the molecular band indices and pseudo-equivalent width for Ca I $4226 \AA$ were determined following the prescriptions in $\S$ II. In general, because of possible continuum slope problems, it is most reliable to use these theoretical indices differentially to estimate relative abundances between different groups of $\omega$ Cen stars. The deduced absolute abundances are least reliable for the $\mathrm{Ca}$ I feature, and more reliable for the molecular features. Some tests of the reliability of synthetic spectra computations of similar indices can be found in Bell (1984).

The solid lines in Figures 1 through 7 represent the predicted feature strengths from synthetic spectra for $Z=1 / 100$ and $1 / 10$ of $Z_{0}$, and, as discussed above, the lowest metallicity group of $\omega$ Cen stars is assumed to have $Z=1 / 100 Z_{0}$, the intermediate metallicity group to have $Z=1 / 30 Z_{0}$, and the highest metallicity group to have $Z=0.1 Z_{0}$. The CNO abundances have been assumed to scale with the overall abundances.

The calculated indices are given in Table 4.

\section{b) Atomic Features}

We look first at the atomic line features. Although there are problems in defining the continuum shape over a $60 \AA$ bandpass in the blue, the behavior of the observed $\mathrm{H}+\mathrm{K} \mathrm{Ca}$ II index in the $\omega$ Cen giants is reproduced reasonably well as a function of $T_{\text {eff }}$ within each metallicity group by the predicted indices from the synthetic spectra. The difference between the low and high abundance groups of $\omega$ Cen stars appears to be reasonably close to the factor of 10 assumed here. The same is true of the $4226 \AA$ feature of $\mathrm{Ca}$ I. The $\mathrm{Ca}$ I line is a better abundance indicator in stars which are more metal-rich than the majority of those discussed here. The zero point shift between the observations and the calculations may be due to the sharpness of the $\mathrm{Ca}$ I line itself (as sampled by $0.4 \AA$ pixels) and to the narrow bandpasses which may not fairly sample the fluctuating background of weaker absorption lines.

The $\mathrm{Ca}$ I index is slightly dependent on the carbon abundance, owing to the $\mathrm{CH}$ lines occurring in the feature and continuum bands, but this effect appears to be too small to explain the effect seen in Figure 7. The continuum depression caused by the $\mathrm{CH}$ and $\mathrm{CN}$ bands in the carbon stars is extremely large (cf Bell and Dickens 1974, Fig. 6), and a completely new calibration is necessary before metallicities can be derived for them.

It is extremely interesting that there is a noticeable tendency for the weak $\mathrm{CO}$ stars to have stronger atomic features than their strong $\mathrm{CO}$ counterparts within each metallicity group. This effect appears in both the $\mathrm{H}+\mathrm{K} \mathrm{Ca}$ II index and the 4226 $\AA \mathrm{Ca}$ I line, and corresponds to an enhancement of about 0.5 dex. We will return to this point later.

\section{c) Molecular Features}

PFCAM deduced that the difference in the $\mathrm{CO}$ indices between $\mathrm{CO}$ strong and $\mathrm{CO}$ weak stars at a fixed $T_{\text {eff }}$ and metallicity corresponds to a $\mathrm{C}$ depletion of $0.8 \mathrm{dex}$, for a fixed $\mathrm{O} / Z$ ratio. A carbon depletion of this magnitude is seen in the most luminous stars in M29 and in NGC 6397. However, if the oxygen abundance is constant, the maximum difference in $\mathrm{C}$ abundance from the lack of an obvious separation in $\mathrm{CH}$ indices between the two groups of stars is $0.4 \mathrm{dex}$, which is not enough to produce the two separate $\mathrm{CO}$ sequences. Frogel, Persson, and Cohen (1981) suggested that the extremely strong violet $\mathrm{CN}$ bands seen in the $\mathrm{CO}$ weak stars may result in the red system $\mathrm{CN}$ bands in the infrared reducing the observed $\mathrm{CO}$ indices by a few hundredths of a magnitude. This very interesting suggestion was based upon inspection of calculations of $\mathrm{CN}$ line absorption in the $2 \mu \mathrm{m}$ region and studies of high-resolution spectra of $\alpha$ Boo and $\beta$ And. It was suggested that the depletion of $\mathrm{C}$ and enhancement of $\mathrm{N}$ may together be adequate to explain the separation of $\mathrm{CO}$ indices. In order to check this hypothesis, we carried out detailed synthetic spectrum and model atmosphere calculations. Models were computed for $[\mathrm{A} / \mathrm{H}]=-1.0$ and $-2.0 \mathrm{dex}$, with the nitrogen abundance enhanced by $1.0 \mathrm{dex}$. Infrared synthetic spectra were then computed for these models, again with the high nitrogen abundance. This increase in $\mathrm{CN}$ does increase the red $\mathrm{CN}$ line absorption in the $\mathrm{CO}$ comparison filter band, to about 0.5 mag for the model $4000 / 0.9 /-1.0$, but a similar increase in 
TABLE 4

COMPUTED INDICES FRom SyNTHETIC SPECTRA

\begin{tabular}{|c|c|c|c|c|c|c|c|c|c|}
\hline $\begin{array}{c}\text { Model } \\
\left(T_{\text {eff }}, \log \mathrm{g},[\mathrm{Fe} / \mathrm{H}]\right)\end{array}$ & $\begin{array}{l}\text { NH } \\
(\%)\end{array}$ & $\begin{array}{c}\mathrm{LoCN} \\
(\%)\end{array}$ & $\begin{array}{c}\mathrm{CN} 38 \\
(\%)\end{array}$ & $\begin{array}{l}\text { CN42 } \\
(\%)\end{array}$ & $\begin{array}{l}\mathrm{CH} \\
(\%)\end{array}$ & $\begin{array}{l}\mathrm{C}_{2} \\
(\%)\end{array}$ & $\begin{array}{c}\mathrm{CO} \\
(\mathrm{mag})\end{array}$ & $\begin{array}{c}\mathrm{Ca} \mathrm{I} \\
(\AA)\end{array}$ & $\begin{array}{c}\mathrm{H}+\mathrm{K} \\
(\%)\end{array}$ \\
\hline \multicolumn{10}{|c|}{ A. Normal C, N, O } \\
\hline $\begin{array}{l}4000,1.25,-0.5 \ldots \ldots \\
4500,2.25,-0.5 \ldots \ldots \\
5000,3.00,-0.5 \ldots \ldots\end{array}$ & $\begin{array}{l}0.183 \\
0.128 \\
0.191\end{array}$ & $\begin{array}{l}+0.627 \\
+0.535 \\
+0.358\end{array}$ & $\begin{array}{l}0.676 \\
0.570 \\
0.403\end{array}$ & $\begin{array}{l}+0.160 \\
+0.130 \\
+0.067\end{array}$ & $\begin{array}{l}0.254 \\
0.265 \\
0.250\end{array}$ & $\begin{array}{l}-0.007 \\
+0.008 \\
+0.010\end{array}$ & $\begin{array}{l}0.172 \\
0.116 \\
0.074\end{array}$ & $\begin{array}{l}6.55 \\
4.48 \\
3.35\end{array}$ & $\begin{array}{l}0.558 \\
0.432 \\
0.424\end{array}$ \\
\hline $\begin{array}{l}4000,0.90,-1.0 \ldots \ldots \\
4500,1.80,-1.0 \ldots \ldots \\
5000,3.00,-1.0 \ldots \ldots\end{array}$ & $\begin{array}{l}0.190 \\
0.229 \\
0.191\end{array}$ & $\begin{array}{l}+0.538 \\
+0.385 \\
+0.206\end{array}$ & $\begin{array}{l}0.590 \\
0.441 \\
0.249\end{array}$ & $\begin{array}{l}+0.116 \\
+0.070 \\
+0.026\end{array}$ & $\begin{array}{l}0.250 \\
0.258 \\
0.243\end{array}$ & $\begin{array}{l}-0.011 \\
-0.003 \\
-0.002\end{array}$ & $\begin{array}{l}0.150 \\
0.100 \\
0.049\end{array}$ & $\begin{array}{l}5.12 \\
3.56 \\
2.69\end{array}$ & $\begin{array}{l}0.503 \\
0.412 \\
0.412\end{array}$ \\
\hline $\begin{array}{l}4000,0.50,-2.0 \ldots \ldots \\
4500,1.50,-2.0 \ldots \ldots \\
5000,2.50,-2.0 \ldots \ldots\end{array}$ & $\begin{array}{l}0.206 \\
0.212 \\
0.130\end{array}$ & $\begin{array}{l}+0.215 \\
+0.060 \\
-0.001\end{array}$ & $\begin{array}{l}0.244 \\
0.114 \\
0.028\end{array}$ & $\begin{array}{l}+0.017 \\
+0.005 \\
+0.004\end{array}$ & $\begin{array}{l}0.210 \\
0.236 \\
0.186\end{array}$ & $\begin{array}{l}-0.012 \\
-0.010 \\
-0.004\end{array}$ & $\begin{array}{l}0.099 \\
0.046 \\
0.013\end{array}$ & $\begin{array}{l}2.53 \\
2.06 \\
1.30\end{array}$ & $\begin{array}{l}0.426 \\
0.409 \\
0.415\end{array}$ \\
\hline \multicolumn{10}{|c|}{ B. $[\mathrm{C}, \mathrm{N}, \mathrm{O} / \mathrm{Fe}]=-0.1,+0.26,0.0 \mathrm{dex}$} \\
\hline $\begin{array}{l}4000,1.25,-0.5 \ldots \ldots \\
4500,2.25,-0.5 \ldots \ldots \\
5000,3.00,-0.5 \ldots \ldots\end{array}$ & $\begin{array}{l}0.173 \\
0.217 \\
0.222\end{array}$ & $\begin{array}{l}+0.654 \\
+0.606 \\
+0.487\end{array}$ & $\begin{array}{l}0.686 \\
0.610 \\
0.499\end{array}$ & $\begin{array}{l}+0.132 \\
+0.130 \\
+0.082\end{array}$ & $\begin{array}{l}0.245 \\
0.258 \\
0.246\end{array}$ & $\begin{array}{l}\cdots \\
\cdots \\
\cdots\end{array}$ & $\begin{array}{l}0.164 \\
0.105 \\
0.064\end{array}$ & & \\
\hline $\begin{array}{l}4000,0.90,-1.0 \ldots \ldots \\
4500,1.80,-1.0 \ldots \ldots \\
5000,3.00,-1.0 \ldots \ldots\end{array}$ & $\begin{array}{l}0.184 \\
0.239 \\
0.230\end{array}$ & $\begin{array}{l}+0.581 \\
+0.484 \\
+0.339\end{array}$ & $\begin{array}{l}0.612 \\
0.506 \\
0.363\end{array}$ & $\begin{array}{l}+0.094 \\
+0.069 \\
+0.032\end{array}$ & $\begin{array}{l}0.237 \\
0.245 \\
0.236\end{array}$ & $\begin{array}{l}\cdots \\
\cdots \\
\cdots\end{array}$ & $\begin{array}{l}0.147 \\
0.093 \\
0.043\end{array}$ & & \\
\hline $\begin{array}{l}4000,0.50,-2.0 \ldots \ldots \\
4500,1.50,-2.0 \ldots \ldots \\
5000,2.50,-2.0 \ldots \ldots\end{array}$ & $\begin{array}{l}0.298 \\
0.312 \\
0.217\end{array}$ & $\begin{array}{l}+0.240 \\
+0.127 \\
+0.012\end{array}$ & $\begin{array}{l}0.249 \\
0.174 \\
0.038\end{array}$ & $\begin{array}{l}+0.006 \\
-0.004 \\
-0.004\end{array}$ & $\begin{array}{l}0.198 \\
0.221 \\
0.166\end{array}$ & $\begin{array}{l}\cdots \\
\cdots \\
\cdots\end{array}$ & $\begin{array}{l}0.095 \\
0.040 \\
0.011\end{array}$ & & \\
\hline \multicolumn{10}{|c|}{ C. $[\mathrm{C}, \mathrm{N}, \mathrm{O} / \mathrm{Fe}]=-0.2,-0.6,-0.1 \mathrm{dex}$} \\
\hline $\begin{array}{l}4000,1.25,-0.5 \ldots \ldots \\
4500,2.25,-0.5 \ldots \ldots \\
5000,3.00,-0.5 \ldots \ldots\end{array}$ & $\begin{array}{l}0.187 \\
0.225 \\
0.239\end{array}$ & $\begin{array}{l}+0.675 \\
+0.652 \\
+0.555\end{array}$ & $\begin{array}{l}0.694 \\
0.639 \\
0.548\end{array}$ & $\begin{array}{l}+0.168 \\
+0.176 \\
+0.126\end{array}$ & $\begin{array}{l}0.245 \\
0.258 \\
0.244\end{array}$ & $\begin{array}{c}\cdots \\
\cdots \\
\cdots \cdots\end{array}$ & $\begin{array}{l}0.152 \\
0.096 \\
0.056\end{array}$ & & \\
\hline $\begin{array}{l}4000,0.90,-1.0 \ldots \ldots \\
4500,1.80,-1.0 \ldots \ldots \\
5000,3.00,-1.0 \ldots \ldots\end{array}$ & $\begin{array}{l}0.200 \\
0.253 \\
0.253\end{array}$ & $\begin{array}{l}+0.616 \\
+0.551 \\
+0.412\end{array}$ & $\begin{array}{l}0.631 \\
0.553 \\
0.424\end{array}$ & $\begin{array}{l}+0.131 \\
+0.110 \\
+0.057\end{array}$ & $\begin{array}{l}0.237 \\
0.243 \\
0.232\end{array}$ & $\begin{array}{l}\cdots \\
\cdots \\
\cdots\end{array}$ & $\begin{array}{l}0.134 \\
0.084 \\
0.037\end{array}$ & & \\
\hline $\begin{array}{l}4000,0.50,-2.0 \ldots \ldots \\
4500,1.50,-2.0 \ldots \ldots \\
5000,2.50,-2.0 \ldots \ldots\end{array}$ & $\begin{array}{l}0.335 \\
0.358 \\
0.287\end{array}$ & $\begin{array}{l}+0.292 \\
+0.198 \\
+0.036 \\
\end{array}$ & $\begin{array}{l}0.296 \\
0.240 \\
0.059 \\
\end{array}$ & $\begin{array}{l}+0.029 \\
+0.010 \\
-0.001\end{array}$ & $\begin{array}{l}0.197 \\
0.216 \\
0.152 \\
\end{array}$ & $\begin{array}{l}\cdots \\
\cdots \\
\cdots\end{array}$ & $\begin{array}{l}0.087 \\
0.034 \\
0.009 \\
\end{array}$ & & \\
\hline \multicolumn{10}{|c|}{ D. Lower Gravity AGB Models } \\
\hline $\begin{array}{l}4500,1.50,-1.0 \ldots \ldots \\
5000,2.25,-1.0 \ldots \ldots\end{array}$ & $\begin{array}{l}0.217 \\
0.182\end{array}$ & $\begin{array}{l}+0.432 \\
+0.207\end{array}$ & $\begin{array}{l}0.473 \\
0.263\end{array}$ & $\begin{array}{l}+0.043 \\
+0.019\end{array}$ & $\begin{array}{l}0.246 \\
0.231\end{array}$ & $\begin{array}{l}\cdots \\
\cdots\end{array}$ & $\begin{array}{l}\cdots \\
\cdots\end{array}$ & & $\begin{array}{l}0.445 \\
0.427\end{array}$ \\
\hline $\begin{array}{l}4500,0.75,-2.0 \ldots \ldots \\
5000,2.25,-2.0 \ldots \ldots\end{array}$ & $\begin{array}{l}0.163 \\
0.129\end{array}$ & $\begin{array}{l}+0.044 \\
+0.008\end{array}$ & $\begin{array}{l}0.098 \\
0.031\end{array}$ & $\begin{array}{l}+0.009 \\
+0.005\end{array}$ & $\begin{array}{l}0.178 \\
0.158\end{array}$ & $\begin{array}{l}\cdots \\
\cdots\end{array}$ & $\begin{array}{l}\cdots \\
\cdots\end{array}$ & & $\begin{array}{l}0.431 \\
0.420\end{array}$ \\
\hline
\end{tabular}

line absorption occurs in the CO filter band itself. These synthetic spectra calculations were made using Spindler's (1965) Franck-Condon factors, which agree quite well with the values found by Sneden and Lambert (1982) for the 0-2 and 1-3 bands in the solar spectrum. Although this hypothesis is not borne out by detailed synthetic spectra calculations for normal giants with very depleted $\mathrm{C}$ and $\mathrm{O}$ and enhanced $\mathrm{N}$, it might be true for $\mathrm{CH}$ stars with their very strong $\mathrm{CN}$ bands.

The NH feature can be discussed completely separately from the carbon features since the primary depletion of nitrogen is $\mathrm{N}_{2}$ and not $\mathrm{CN}$. The $\mathrm{NH}$ feature shows a strong separation between the $\mathrm{CO}$ strong and $\mathrm{CO}$ weak $\omega$ Cen giants. The $\mathrm{CO}$ strong stars appear to have $[\mathrm{N} / Z]=0$. This separation implies an enhancement of at least a factor of 10 in $N / Z$ (at all metallicities) in the $\mathrm{CO}$ weak giants. The greatest values of the NH index are found for the enhanced nitrogen models with $[M / \mathrm{H}]=-2.0$ dex, owing to these models having the lowest line blanketing in the comparison bandpasses.

The $\mathrm{CH}$ feature is predicted quite well as a function of $T_{\text {eff }}$ by the synthetic spectra. The observed $\mathrm{CH}$ indices imply that, if
$[\mathrm{O} / Z]=0.0,[\mathrm{C} / Z]$ is between 0.2 and -0.5 dex in each of the metallicity groups, and there is no obvious separation between the $\mathrm{CO}$ strong and $\mathrm{CO}$ weak stars. However, in view of the existence in the $\mathrm{CO}, \mathrm{CN}$, and $\mathrm{NH}$ features of a clear separation of band strengths between the $\mathrm{CO}$ weak and the $\mathrm{CO}$ strong stars and the lack of such in the $\mathrm{CH}$ band, it is necessary to ask if we can find values of the $\mathrm{C}$ and $\mathrm{O}$ abundances which give the observed $\mathrm{CH}$ band strengths and simultaneously give the $\mathrm{N}$ abundance found from the NH bands. Clearly, there is an ambiguity in the carbon abundance found from $\mathrm{CH}$ in the absence of knowledge of the oxygen abundance. The lower the oxygen abundance, the lower the carbon abundance can be to match the $\mathrm{CH}$ features, since less $\mathrm{C}$ is lost via $\mathrm{CO}$ formation. Enhancement of nitrogen by 1 dex corresponds to depletion of both $\mathrm{C}$ and $\mathrm{O}$ by 0.7 dex. We consequently computed synthetic spectra using these abundances.

The $4200 \AA \mathrm{CN}$ index is matched reasonably well in the $\mathrm{CO}$ strong stars by $\mathrm{C} / Z \times \mathrm{N} / Z$ ratios of 1 to 3 . However, in the $\mathrm{CO}$ weak stars, the $4200 \AA \mathrm{CN}$ band is extremely strong. Given that the $\mathrm{C}$ abundance is constrained by the $\mathrm{CH}$ indices, 
an increase in the ratio of $\mathrm{N} / Z$ of between 10 and 30 at all metallicities is derived from the fits to the $\mathrm{CN}$ observations of the $\mathrm{CO}$ weak giants in $\omega \mathrm{Cen}$. This nitrogen enhancement is in excellent agreement with the $\mathrm{N}$ enhancements seen in $\mathrm{NH}$ and found earlier (Dickens and Bell 1976).

The behavior of the $3880 \AA \mathrm{CN}$ band shown in Figure $4 a-c$ is similar. A separation of about a factor of 10 in $N / Z$ exists between the low $\mathrm{CO}$, strong $\mathrm{CN}$ stars, and the strong $\mathrm{CO}$, normal $\mathrm{CN}$ stars. The absolute normalization is correct for the low and intermediate metallicity groups in that the mean strength of the $3883 \AA \mathrm{CN}$ band is close to that predicted for $\mathrm{C} / Z$ and $\mathrm{N} / Z=1$. In the highest metallicity group of $\omega$ Cen giants, the observed molecular indices for the $\mathrm{CO}$ strong stars are somewhat below those predicted for $\mathrm{N} / Z$ and $\mathrm{C} / Z=1$; the separation in $\mathrm{CN}$ between the $\mathrm{CO}$ weak and $\mathrm{CO}$ strong stars is still clear.

\section{d) Summary of $\mathrm{C}$ and $\mathrm{N}$ Abundances}

There are three groups of $\omega$ Cen giants: the four carbon stars; the group of stars with weak $\mathrm{CO}$, strong $\mathrm{NH}$, and strong $\mathrm{CN}$; and the group of stars with strong $\mathrm{CO}$, weak $\mathrm{NH}$, and weak $\mathrm{CN}$ for their metallicities. The atomic and molecular lines are consistent with the weak $\mathrm{CO}$ stars having depleted carbon and oxygen, $\mathrm{N} / Z=10$ to 30 , and heavy element metallicities $(Z)$ which are 3 times larger than the strong $C O$ stars at a given position in the $H-R$ diagram. We denote these two groups as the $\mathrm{N}$-enhanced and $\mathrm{N}$-normal stars. If $Z$ of the $\mathrm{N}$-enhanced stars is actually higher, $\mathrm{C} / Z=\frac{1}{4}$ to $\frac{1}{2}$ and $\mathrm{N} /$ $Z=5-15$ among this group.

\section{DISCUSSION}

\section{a) The N-Enhancement Problem}

In the previous section, we demonstrated that there are two groups of giants in $\omega \mathrm{Cen}$, the $\mathrm{N}$-enhanced and the $\mathrm{N}$-normal giants, as well as four carbon stars. In order to produce the $\mathrm{N}$-enhanced stars from the $\mathrm{N}$-normal stars, it is necessary that both $\mathrm{C}$ and $\mathrm{O}$ be depleted. Note that this hypothesis requires that the $\left[\mathrm{O}_{\mathrm{I}}\right]$ lines in the spectra of such stars as RGO 253 should be invisible, consistent with the upper limit of $25 \mathrm{~m} \AA$ of Cohen (1981). If the oxygen abundance is constant, the $\mathrm{CH}$ indices permit a maximum range in $\mathrm{C}$ abundance of a factor of 2. If one assumes $\mathrm{C} / \mathrm{N}$ in the unmixed stars to be twice solar and that half of the $\mathrm{C}$ is burned to $\mathrm{N}$, a nitrogen enhancement factor of 6 can be obtained. But some of the N-enhanced stars have $\mathrm{N}$ augmented by a factor of 15 ; this high degree of $\mathrm{N}$ enhancement cannot be produced by $\mathrm{CN}$ burning alone, but must involve $\mathrm{ON}$ burning and subsequent mixing (see Sweigart and Mengel 1979). For the most extreme N-enhanced stars, it may not be possible to burn $\mathrm{C}$ and $\mathrm{O}$ into $\mathrm{N}$ in adequate quantities without excessively weakening the $\mathrm{CN}$ bands below those observed. This problem of an excessive degree of $\mathrm{N}$ enhancement is not unique to $\omega \mathrm{Cen}$; it has been found in chemically homogeneous globular clusters such as NGC 1851 (Hesser et al. 1982), which contains stars apparently identical to RGO 253, and in M92 and M15 (see the extensive discussion in Carbon et al. 1982; Trefzger et al. 1983; Suntzeff 1981).

\section{b) The Ca Enhancement Problem}

In $\S$ IV we have shown that the $\mathrm{N}$-enhanced $\omega$ Cen giants also apparently have $\mathrm{Ca}$ abundances a factor of 3 higher than the $\mathrm{N}$-normal stars with the same position in the H-R diagram. It is possible that these stars represent the more metal-rich ones within each metallicity group. But this then suggests that a slight heavy metal enhancement greatly increases the probability of mixing at a given luminosity (which may also depend on the rotation rate of the stellar core). Given the presence of a significant fraction of unmixed stars even in the highest metallicity group, this cannot be true. Is the slight $\mathrm{Ca}$ enhancement produced during the mixing? All calculations of stellar evolution vehemently deny this, but mixing calculations are notoriously difficult and have not met the test of detailed confrontation with observations well in the past. It is also possible that the very strong $\mathrm{CN}$ bands in the $\mathrm{N}$-enhanced stars have affected the continuum placement in these moderate-resolution spectra. Our carbon star spectra clearly show such effects, and only higher resolution data can eliminate this possibility for the less extreme cases.

Another hypothesis is that the apparent $\mathrm{Ca}$ enhancement in the $\mathrm{N}$-enhanced stars is not real and is due to our inability to separate asymptotic giant branch (AGB) from first-ascent red giants in $\omega$ Cen. Because of the range in heavy element metallicity from star to star within $\omega$ Cen, AGB stars cannot be picked out via their offset from the first-ascent giants in a color magnitude diagram. Thus AGB stars, which will be systematically bluer in the H-R diagram than normal first-ascent GB stars, will have their $R(V-K)$ indices underestimated by 0.15 to 0.3 , based on the large collection of globular cluster photometry by Frogel, Persson, and Cohen (1983). They will therefore appear to have systematically enhanced element line strengths compared to the normal GB stars in the same range of $R(V-K)$.

The AGB stars will also have lower surface gravities than the GB stars due to mass loss during the He flash and to the luminosity difference between the AGB and the RGB. The surface gravity difference at a fixed $T_{\text {eff }}$ can be as large as 0.5 dex. Those features which, in the relevant range of $T_{\text {eff }}$, become stronger for lower gravities, such as $\mathrm{H}+\mathrm{K}$ of $\mathrm{Ca}$ II (see Bell 1985; Suntzeff 1981), will be enhanced in the AGB stars: the enhancements shown in Table 4 are not very large but may be adequate.

The suggested identification of the $\mathrm{N}$-enhanced stars as mixed AGB giants eliminates the apparent heavy element ehancement, and it solves the problem of when doe the mixing event take place (i.e., during the He flash). The detailed abundance analyses of $\omega$ Cen giants such as those of Cohen (1981) do not yet cover a large enough sample to decide whether the apparent $\mathrm{Ca}$ enhancements seen in our moderate-resolution data are real. Cohen (1981) found a general large enhancement of $\mathrm{Ca} / \mathrm{Fe}$, as is typical of metal-poor halo stars. Her mean $[\mathrm{Ca} / \mathrm{Fe}]$ of 0.7 dex may be an overestimate, since the very precise calcium oscillator strengths of Smith and Raggett (1981) are, on average, 0.18 dex larger than the values she used. Cohen found a range in $\mathrm{Ca} / \mathrm{Fe}$ from star to star within $\omega$ Cen of about a factor of 2 , but whether this is dominated by errors in the analysis and whether the increase in $\mathrm{Ca} / \mathrm{Fe}$ correlates with $\mathrm{N}$ enhancement is not yet clear.

Is the suggested identification of the $\mathrm{N}$-enhanced stars as AGB stars reasonable based on their relative numbers? The Cannon and Stobie (1973) unbiased sample of confirmed proper motion $\omega$ Cen giants with $B-V>0.85 \mathrm{mag}$ and $11<V<13 \mathrm{mag}$ consists of 47 stars, $60 \%$ of which were observed by PFCAM and also are included here. All the reddest stars $(B-V>1.4 \mathrm{mag})$ in the unbiased Cannon and 
Stobie sample were included here; the fraction of very red stars $(B-V>1.5 \mathrm{mag})$ in the unbiased survey is less than $10 \%$ of the total, but is about $40 \%$ of the present sample. Thus there is tendency to skew the abundance distribution toward higher abundance $\omega$ Cen stars [i.e., those with large values of $R(V-K)]$. However, the small difference in luminosity between first and second ascent giant branch stars at a given color should lead to a sample essentially unbiased in its ratio of GB to AGB stars. About $40 \%$ of the unbiased sample where discrimination is possible belong to the $\mathrm{CO}$ weak, $\mathrm{N}$-enhanced stars. This is a reasonable fraction for the ratio of second to first-ascent red giants near the red giant tip, although the expected fraction falls rapidly as one descends in luminosity down the giant branch (see Green 1981). Selection effects in our sample preclude use of a luminosity function to further test this hypothesis.

\section{c) Comparison with Other Clusters}

There is a large body of data on $\mathrm{CN}$ and $\mathrm{CH}$ variations among the "chemically homogeneous" globular clusters. The existence of a range of $\mathrm{CN}$ band strengths or a bimodel distribution of $\mathrm{CN}$ band strengths at a given $T_{\text {eff }}$, has been widely noted (see, for example, Dickens, Bell, and Gustafsson 1979; Norris and Smith 1983; Smith and Norris 1983 and references therein, as well as the work of the Lick group cited earlier in $\S \mathrm{Va}$ ). The much smaller range in $\mathrm{CH}$ band variations than in the variation in strength of the $3883 \AA \mathrm{CN}$ band is also a common theme of these studies. The main difference at first sight is that the $\mathrm{N}$ enhancements in many clusters are claimed to be independent of whether a star is a first or second-ascent red giant, and of how close to the giant branch tip it may be. In $\omega$ Cen, it is still plausible that the $\mathrm{N}$-enhanced stars are AGB stars.

AGB stars are expected to reside on the blue side of the giant branch. The N-enhanced stars in NGC 1851 (Hesser et al. 1982) show no tendency to do so. Nor is such an effect seen within each metallicity group of $\omega$ Cen giants. Thus the hypothesis presented in $\S \mathrm{V} b$ that the $\mathrm{N}$-enhanced stars in $\omega$ Cen are AGB giants may not in fact be correct. Analogy to other globular clusters such as M92 (Carbon et al. 1982) (see also $\S \mathrm{V} c$ ) where such $\mathrm{N}$-enhanced stars have been seen on the subgiant branch below the minimum luminosity of the AGB also suggests that the N-enhanced stars are not all AGB stars. Lower luminosity stars in $\omega$ Cen and in other globular clusters must be studied to understand the origin of the $\mathrm{N}$-enhanced stars.

There have been several claims of correlations of enhanced $\mathrm{Ca}$ with enhanced $\mathrm{CN}$ in clusters traditionally viewed as chemically homogeneous. These are summarized in Norris and Freeman's (1983) extensive analysis of about 100 red giants in $\mathrm{M} 22$. They find a wide range in $\mathrm{CN}$ strength and also a range in $\mathrm{H}+\mathrm{K} \mathrm{Ca}$ II line strength, correlated with $\mathrm{CN}$, at a given color. They assert that their M22 sample has fewer than $10 \%$ AGB stars, based on the ratio of AGB stars to red giants at magnitudes just above the horizontal branch where the first and second-ascent red giants are well separated in H-R diagrams. However, the luminosity function for first and secondascent red giants is very different (see, for example, Iben and Truran 1978), and substantial AGB contamination at higher luminosities can be expected.

A clear separation of first-ascent red giants from AGB stars is particularly difficult in M22 because of the large reddening and its spatial variation (see Cohen 1981) over the face of the cluster. This produces an H-R diagram whose sequences are neither tight nor cleanly separated. Could the N-enhanced, $\mathrm{Ca}$ rich M22 stars be AGB giants and the Ca enhancement be only an artifact? The $\mathrm{N}$-enhanced stars in M22 again tend to be on the red side of the giant branch, which is the opposite of that expected for AGB stars.

\section{d) The C Stars}

The five $\mathrm{C}$ stars known to be members of $\omega$ Cen require a separate mixing event from the $\mathrm{N}$-enhanced giants. Since all the carbon stars are brighter than the horizontal branch, one may assume they are AGB stars which have undergone mixing of $\mathrm{C}$ formed by triple- $\alpha$ reactions. Their luminosities are too low for thermal pulses to have occurred, and no satisfactory theoretical explanation exists for carbon stars of such low luminosity. Two C stars are also known in M22 (McClure and Norris 1977; Hesser and Harris 1979) and one in M55 (LloydEvans 1979). It is not yet possible to establish why only certain clusters have $\mathrm{C}$ stars. The cluster mass or metallicity are not the determining factor. (See McClure 1979 for further discussion of these objects.)

\section{SUMMARY}

Spectral scans of 72 stars in $\omega$ Cen have been used to measure the strengths of molecular bands of $\mathrm{CH}, \mathrm{NH}$, and $\mathrm{CN}$, as well as atomic lines of $\mathrm{Ca}$ I and $\mathrm{Ca}$ II. These indices have been combined with the published $\mathrm{CO}$ indices and infrared colors of PFCAM and calibrated using identical indices computed from spectra synthesized from model atmospheres of the appropriate $T_{\text {eff }}$ and surface gravities using reasonably complete line lists. Because $\omega$ Cen has a wide range in heavy element metallicity, the analysis is somewhat complicated. We use a parameter $R(V-K)$ introduced by PFCAM which is based on the position of a star in the H-R diagram with respect to the loci of giants in chemically homogeneous, well-studied globular clusters to separate the $\omega$ Cen giants into lowest, intermediate, and highest metallicity groups.

We find (in addition to the four $C$ stars) two groups of $\omega$ Cen giants. The $\mathrm{N}$-enhanced group has $\mathrm{N} / Z$ enhanced by a factor of 5 to 15 , while $C$ may be depleted by a factor not larger than 4 with respect to the $\mathrm{N}$-normal group, which has $\mathrm{C} / Z$ and $\mathrm{N} / Z$ solar. The $\mathrm{N}$-enhanced stars also have weaker $2.4 \mu \mathrm{m} \mathrm{CO}$ indices and stronger atomic $\mathrm{Ca}$ features than the $\mathrm{N}$-normal stars of the same metallicity group and $T_{\text {eff }}$. The very large nitrogen enhancements seen in some of the $\omega$ Cen giants require processing of both $\mathrm{C}$ and $\mathrm{O}$ into $\mathrm{N}$.

Once the effects of the spread in heavy element metallicity within $\omega$ Cen have been removed, the pattern of the variations of the light elements within $\omega$ Cen is similar to that seen in other globular clusters.

J. G. C. is grateful to NSF grant AST 82-12270 for support. The work of R. A. B. on this project was supported by both funds and supercomputer time from NSF grant AST 80-19570 after $9 / 1 / 84$. The indices were computed using a Cray I-S of Boeing Computing Services, the University of Maryland Computer Science Center's Univac 1182, and a Ridge $32 \mathrm{C}$. The assistance of Mr. J. Ohlmacher with the calculations is gratefully acknowledged. 
Bell, R. A. 1984, Pub. A.S.P., 96, 318 1985, Pub. A.S.P., 97, 219.

Bell, R. A., and Dickens, R. J. 1974, M.N.R.A.S., 166, 89 1980, Ap. J., 242, 657

Bell, R. A., Dickens, R. J., and Gustafsson, B. 1979, Ap. J., 282, 428.

Bell, R. A. and Gustafsson, B. 1978, Astr. Ap. Suppl., 34, 229. 1983, M.N.R.A.S., 204, 249

Bond, H. E. 1975, Ap. J. (Letters), 202, L47.

Cannon, R. D., and Stobie, R.S. 1973, M.N.R.A.S., 162, 207.

Carbon, D. F., et al. 1982, Ap. J. Suppl., 49, 207.

Cohen, J. G. 1978, Ap. J., 223, 487. 1981, Ap.J., 247, 869.

- 1983, Ap. J., 270, 654.

Dickens, R. J. 1972, M.N.R.A.S., 159, 7P

Dickens, R. J., and Bell, R. A. 1976, Ap. J., 207, 506.

Dickens, R. J., Bell, R. A., and Gustafsson, B. 1979, Ap. J., 232, 428.

Freeman, K. C., and Rodgers, A. W. 1975, Ap. J. (Letters), 201, L71.

Frogel, J. A., Persson, S.E., and Cohen, J.G. 1981, Ap. J., 246, 842. . 1983, Ap. J. Suppl., 53, 713.

Gratton, R. 1982, Astr. Ap., 115, 336

Green, E. 1981, Ph.D. thesis, University of Texas at Austin.

Harding, G. A. 1972, Observatory, 82, 205.

Hesser, J. E., Bell, R. A., Cannon, R. D., and Harris, G. L. H. 1982, A.J., 87, 1470.

1985, Ap. J., 295, 437.

Hesser, J. E., and Harris, G. L. H. 1979, Ap. J., 234, 513.

\section{REFERENCES}

Iben, I., Jr., and Truran, J. W. 1978, Ap. J., 220, 980.

Lambert, D. L. 1978, M.N.R.A.S., 182, 249.

Lloyd Evans, T. 1979, private communication. 1983, M.N.R.A.S., 204, 975 .

Mallia, E. A., and Pagel, B. E. J. 1981, M.N.R.A.S., 194, 421

McClure, R. D. 1979, Mem. Soc. Astr. Italiana, 50, 15.

McClure, R. D., and Norris, J. 1977, Ap. J. (Letters), 217, L101.

Norris, J., and Bessell, M. S. 1975, Ap. J. (Letters), 201, L75.

Norris, J., and Freeman, K. C. 1983, Ap. J., 266, 130.

Norris, J., and Smith, G. H. 1983, Ap. J., 275, 120.

Persson, S. E., Frogel, J. A., Cohen, J. G., Aaronson, M., and Matthews, K. 1980, Ap. J., 235, 452 (PFCAM).

Shectman, S. 1978, Carnegie Institution of Washington Year Book, 77, 200.

Smith, G., and Ragett, D. St. J. 1981, J. Phys. B., 14, 4015.

Smith, G. H., and Norris, J. 1983, Ap. J., 264, 215.

Sneden, C., and Lambert, D. L. 1982, Ap. J., 259, 381.

Spindler, J. 1965, J. Quant. Spectrosc. Rad. Transfer, 5, 165.

Suntzeff, N. B. 1980, A.J., 85, 408.

. 1981, Ap. J. Suppl., 47, 1.

Sweigart, A. V., and Mengel, J. G. 1979, Ap. J., 229, 624.

Tomkin, J., and Lambert, D. L. 1984, Ap. J., 279, 220.

Trefzger, C. G., Carbon, D. F., Langer, G. E., Suntzeff, N. B., and Kraft, R. P. 1983, Ap. J., 266, 144.

Webbink, R. F. 1981, Ap. J. Suppl., 45, 259.

Woolley, R. V. d. R. et al. 1966, Royal Obs. Ann., No. 2.

Roger A. Bell: Astronomy Program, University of Maryland, Space Science Building, College Park, MD 20742

Judith G. CoHEN: 105-24, California Institute of Technology, Pasadena, CA 91125 